\title{
Asymptotic Dynamics, Non-Critical and Critical Fluctuations for a Geometric Long-Range Interacting Model
}

\author{
F. Comets ${ }^{1}$ and Th. Eisele ${ }^{2 \star}$ \\ ${ }^{1}$ Université de Paris-Sud, Laboratoire de Stratistique Appliquée UA CNRS 743, Mathématique, Bât. \\ 425, F-91405 Orsay Cédex, France, \\ ${ }^{2}$ Universität Heidelberg, Institut für Angewandte Mathematik, Im Neuenheimer Feld 294, D-6900 \\ Heidelberg 1, Federal Republic of Germany
}

\begin{abstract}
We study the dynamics of geometric spin system on the torus with long-range interaction. As the number of particles goes to infinity, the process converges to a deterministic, dynamical magnetization field that satisfies an Euler equation (law of large numbers). Its stable steady states are related to the limits of the equilibrium measures (Gibbs states) of the finite particle system. A related equation holds for the magnetization densities, for which the property of propagation of chaos also is established. We prove a dynamical central limit theorem with an infinite-dimensional Ornstein-Uhlenbeck process as a limiting fluctuation process. At the critical temperature of a ferromagnetic phase transition, both a tighter quantity scaling and a time scaling is required to obtain convergence to a one-dimensional critical fluctuation process with constant magnetization fields, which has a non-Gaussian invariant distribution. Similarly, at the phase transition to an antiferromagnetic state with frequency $p_{0}$, the fluctuation process with critical scaling converges to a two-dimensional critical fluctuation process, which consists of fields with frequency $p_{0}$ and has a nonGaussian invariant distribution on these fields. Finally, we compute the critical fluctuation process in the infinite particle limit at a triple point, where a ferromagnetic and an antiferromagnetic phase transition coincide.
\end{abstract}

\section{Introduction}

In this paper, we study the nonequilibrium behaviour of a geometric spin model with weak interaction in the infinite particle limit. For finite $n \in \mathbb{N}$, the $n$-particle model consists of particles located at the sites $0,1 / n, \ldots, n-1 / n$ of the unit circle $\mathbb{T}=\mathbb{R} \bmod \mathbb{Z}$. A one-dimensional spin value $\sigma(i / n)$ is associated to each particle, and the spins interact via a mean-field potential depending on the distance between the particles.

\footnotetext{
* Work supported by Deutsche Forschungsgemeinschaft
} 
In the equilibrium theory, the thermodynamic limit of these geometric models has been studied recently $[7,2]$, and has shown a variety of interesting phase transitions. Depending on the parameters, there exist phase transitions to ferromagnetic states with constant magnetization or transitions to antiferromagnetic states with wave-like magnetization functions of any frequency p. Moreover, secondary phase transitions of first-order occur too (see e.g. the phase diagram in [6]). We find metastable states near these secondary phase transitions. The nucleation behaviour of the system can be described, as it switches from one (meta-) stable state to another stable one ([1]).

Here, however, we are interested in the dynamical laws of these models. We start with a Glauber-type dynamics ([11]) for the $n$-particle system, where the spins flip from time to time to another value with a jump intensity depending on the gradient of the Hamiltonian felt by the particle. Next we establish the asymptotic dynamics of the magnetization field in the infinite particle limit (Euler equation). We obtain a similar equation for the density field of the magnetization and show that a propagation of chaos result holds. Our main results are the infinite particle limits of the non-critical fluctuation process and at the critical fluctuations, which besides an appropriate scaling of the spin values - require a rescaling of the time in order to keep track with the stiffness and long time fluctuations of the critical structure (critical slowing down). As a result, only the critical structure survives the critical scaling, and in the limit, the critical fluctuation process is a low dimensional process (of the dimension of the null space of the infinitesimal operator at the critical point), in contrast to the infinite dimensional non-critical fluctuation process. In fact, the critical fluctuations are of dimension 1 at the critical point of a ferromagnetic phase transition, while they are of dimension 2 at an antiferromagnetic phase transition, and of dimension 3 at a ferro $\sim$ - antiferromagnetic triple point.

Asymptotic dynamics, propagation of chaos results and non-critical fluctuation processes for weakly interacting systems have been extensively studied (see e.g. $[17,21-23,25,26]$, to mention just a few). Dawson [3] also obtained a critical fluctuation process of dimension 1 . All these models have a space-independent weak interaction, and therefore lack a rich structure of phase transitions. In a recent paper, Fritz obtained the Euler equation for a continuous spin model on a lattice with nearest neighbour interaction [10].

We are now going to describe our model and the results of the different sections in more detail. For simplicity, we restrict ourselves here to the case of one space dimension $(d=1)$, though all the results in the later sections are formulated for arbitrary dimension $d$.

For the system consisting of $n$ particles, located at the point of the lattice $\mathbb{T}_{n}=\{i / n, i=0, \ldots, n-1\}$, a spin configuration $\sigma^{n}=n^{-1} \sum_{x \in \mathbb{\pi}_{n}} \sigma(x) \delta_{x}$ has the internal energy

$$
\begin{aligned}
H^{n}\left(\sigma^{n}\right) & =-1 / 2 n \sum_{i, j=1}^{n} \tilde{\mathscr{J}}[(i-j) / n] \sigma(i / n) \sigma(j / n) \\
& =-n / 2 \iint_{\mathbb{T}_{n}^{2}} \tilde{\mathscr{J}}(x-y) \sigma^{n}(d x) \sigma^{n}(d y)=-n / 2\left\langle\sigma^{n}, \tilde{\mathscr{J}} * \sigma^{n}\right\rangle=n H\left(\sigma^{n}\right) .
\end{aligned}
$$


Here, the intensity of the interaction between particles at sites $i / n$ and $j / n$ is $\tilde{\mathscr{J}}[(i-j) / n]$, with $\tilde{\mathcal{J}}$ a smooth real function on $\mathbb{T}$; it depends on the distance between sites: this is a mean-field model, but it possesses enough geometry for showing rich behaviour. Here also, $\delta_{x}$ is the Dirac mass at $x$ and $*$ denotes convolution. The single spin distribution, denoted by $\rho$, is a probability measure on $\mathbb{R}$ with compact support. (Only in the last sections of the paper, when we deal with the specific situation at the critical point of a phase transition, do we impose further conditions on $\rho$ ). The dynamical process of the $n$-particle system is a spin-flip process where the intensity of flipping the spin $\sigma(x)$ at $x \in \mathbb{T}_{n}$ to the new spin value $m$, is equivalent for large $n$ to

$$
-\beta m \partial / \partial \sigma(x) H\left(\sigma^{n}\right)=\beta m \cdot \mathscr{J} * \sigma^{n}(x)
$$

with $\beta>0$ as the inverse temperature, and $\mathscr{J}(x)=(\tilde{\mathscr{I}}(x)+\tilde{\mathscr{I}}(-x)) / 2$. More precisely, the infinitesimal generator $L^{n}$ of the process is

$$
L^{n} f(\sigma)=\sum_{x \in \mathbb{\pi}_{n}} \int\left[f\left(\sigma||_{x}^{n} m\right)-f(\sigma)\right] \exp \left\{\beta m \mathscr{F} * \sigma^{n}(x)-\beta \mathscr{f}(0) \sigma(x)[\sigma(x)+m] / 2 n\right\} \rho(d m),
$$

where $f$ is a continuous function on the spin configuration space and $\sigma \mid{ }_{x}^{n} m$ is the flipped configuration which is equal to $\sigma$ except at $x$, where its value is $m$. It is easy to check that the unique invariant distribution for the infinitesimal generator $L^{n}$ is the $n$-particle Gibbs measure $Q^{n}$ with the Hamiltonian $H^{n}$, given by

$$
Q^{n}\left(d \sigma^{n}\right)=\exp \left\{-\beta H^{n}\left(\sigma^{n}\right)\right\} \prod_{x \in \mathbb{T}_{n}} \rho(d \sigma(x)) / Z^{n},
$$

with normalizing constant $Z^{n}$. $Q^{n}$ lives on the $n$-particle configuration space, which is a closed subset of the set $\mathscr{M}$ of bounded (with respect to the total variation norm) Radon measures endowed with the weak-* topology. The cumulant generating function of the single spin distribution $\rho$ is defined by

$$
\gamma(r)=\log \int_{\mathbb{R}} \exp (r m) \rho(d m) .
$$

Now, we can state the asymptotic dynamics of the spin-flip processes $\sigma_{t}^{n}$, generated by $L^{n}$, in the infinite particle limit.

Theorem 1'. The processes $\sigma_{t}^{n}$ converge in law on the Skorokhod space $\mathscr{D}([0, T], \mathscr{M})$ to the magnetization process $u_{t} \lambda$, where $\lambda$ is the Lebesgue measure on $\mathbb{T}$ and the density $u_{t} \in L^{\infty}(\mathbb{T})$ satisfies the deterministic evolution equation

$$
d / d t u_{t}(x)=\exp \left\{\gamma\left(\beta \mathscr{J} * u_{t}\right)\right\}\left[\gamma^{\prime}\left(\beta \mathscr{J} * u_{t}\right)-u_{t}\right] .
$$

As is to be expected, there is a close connection between (1.6) and the Gibbs states $Q^{n}$. Indeed, it has been shown in [7] that the $Q^{n}$ satisfy a large deviation principle on $\mathscr{M}$ with a rate function

with

$$
V(\mu)=I(\mu)+\beta H(\mu)
$$

$$
I(\mu)=\left\{\begin{array}{cc}
\int_{\bar{\tau}} i(d \mu / d \lambda(x)) \lambda(d x) & \text { if } \mu \ll \lambda, \\
+\infty & \text { otherwise }
\end{array}\right.
$$


where

$$
i(q)=\sup _{r \in \mathbb{R}}\{q \cdot r-\gamma(r)\}
$$

is the Cramer transform of $\rho$. The large deviation principle means heuristically that for a small weak-* neighborhood $U(\mu)$ of $\mu \in \mathscr{M}$

$$
\text { " } Q^{n}(U(\mu)) \text { behaves asymptotically like } \exp \{-n[V(\mu)-\inf V(v)]\} . "
$$

But the Frechet derivative of $u \mapsto V(u \lambda)$ in the $\|\cdot\|_{\infty}$-norm is by (1.8) and (1.2)

$$
\nabla V(u \lambda)(x)=i^{\prime}(u(x))-\beta \mathscr{J} * u(x)=\left(\gamma^{\prime}\right)^{-1}(u(x))-\beta \mathscr{J} * u(x),
$$

but if $\gamma^{\prime}(0)=0$, i.e. $\rho$ has mean zero,

$$
\operatorname{sign}\left(\gamma^{\prime}(\beta \mathscr{J} * u(x))-u(x)\right)=\operatorname{sign}(-\nabla V(u \lambda)(x))
$$

since $i^{\prime}$ is the inverse of $\gamma^{\prime}$ by (1.9), and since $\gamma^{\prime}(0)=0$ implies $\operatorname{sign} \gamma^{\prime}(r)=\operatorname{sign}(r)$. This means that the right-hand side of the evolution equation has the same sign as $-\nabla V(u \lambda)$. In particular, its paths go downhill with respect to the potential $V$, and the stable steady state solutions of (1.6) are exactly the local minima of $V$.

In Sect. 4, we study the asymptotic dynamics of the density process

$$
\pi_{t}^{n}=n^{-1} \sum_{x \in \mathbb{T}_{n}} \delta_{\left(\sigma_{t}^{n}(x), x\right)},
$$

which is a probability measure on $\mathbb{R} \times \mathbb{T}$. Again we give the space $\mathscr{P}(\mathbb{R} \times \mathbb{T})$ of all such probability measures the weak-* topology. Notice that since for each $x \in \mathbb{T}_{n}, \pi_{t}^{n}(d m,\{x\})=n^{-1} \delta_{\left(\sigma_{t}^{n}(x)\right)}$ is a one-point measure on $\mathbb{R}, \pi^{n}$ and $\sigma^{n}$ contain mathematically the same information. This is however no longer true in the infinite particle limit.

Theorem 2'. $\pi_{t}^{n}$ converges in law to the magnetization density process $h_{t}(m, x) \rho(d m) \lambda(d x)$, where $h_{t}$ satisfies the deterministic density evolution equation:

$$
d / d t h_{t}(m, x)=\exp \left\{m \beta \mathscr{J} * u_{t}(x)\right\}-h_{t}(m, x) \exp \left\{\gamma\left(\beta \mathscr{J} * u_{t}(x)\right)\right\} .
$$

Equation (1.14) is a disintegrated version of (1.6). In fact, multiplying both sides of (1.14) with $m$ and integrating with respect to $\rho(\mathrm{dm})$ gives exactly (1.6). In a similar way, we define the higher order correlation densities for different sites of $\pi$. It is then easy to show that in the infinite particle limit, these correlation densities satisfy a propagation of chaos property. (See Theorems 3 and 3 bis of Sect. 4 for details).

Next, we look for a first order approximation to $u_{t}$; we define the (non-critical) fluctuation process

$$
\zeta^{n t}=n^{1 / 2}\left(\sigma_{t}^{n}-u_{t} \lambda\right)
$$

In order to establish a central limit theorem for these fluctuation processes, we have not only to work in the space $\varphi^{\prime}$ of distributions on $\mathbb{T}$, or at least in a Sobolev space $H_{r_{0}}$ with sufficiently low negative index (see Sect. 5 for technical details), but we also need first a law of large number results for the second moment magnetization fields

$$
\left(\sigma_{t}^{n}\right)^{2}=n^{-1} \sum_{x \in \mathbb{T}_{n}} \sigma_{t}^{2}(x) \delta_{x}
$$


From Theorem $2^{\prime}$, we see that $\left(\sigma_{t}^{n}\right)^{2}$ converge in law on $\mathscr{D}([0, T], \mathscr{M})$ to the second moment magnetization process $v_{t} \lambda$, where $v_{t}$ satisfies the deterministic equation

$$
d / d t v_{t}=\exp \left\{\gamma\left(\beta \mathscr{J} * u_{t}\right)\right\}\left[\gamma^{\prime \prime}\left(\beta \mathscr{J} * u_{t}\right)+\left(\gamma^{\prime}\right)^{2}\left(\beta \mathscr{J} * u_{t}\right)-v_{t}\right]
$$

with $u_{t}$ from (1.6). Now, we can state the central limit theorem for the fluctuation process:

Theorem 4'. If $\mathscr{J}$ is sufficiently smooth and $\zeta_{0}^{n}$ converges in a Sobolev sense to some $\zeta_{0} \in \mathscr{S}^{\prime}$, then the processes $\zeta_{t}^{n}$ converge in law to a $\mathscr{S}^{\prime}$-valued diffusion process $\zeta_{t}$, given by

$$
\begin{aligned}
d \zeta_{t}= & -\gamma^{\prime \prime}\left(\beta \mathscr{J} * u_{t}\right) \exp \gamma\left(\beta \mathscr{J} * u_{t}\right) d^{2} V\left(u_{t}\right) \zeta_{t} d t \\
& +\left[\exp \gamma\left(\beta \mathscr{J} * u_{t}\right)\left(\gamma^{\prime \prime}\left(\beta \mathscr{J} * u_{t}\right)+\left(\gamma^{\prime}\right)^{2}\left(\beta \mathscr{J} * u_{t}\right)-2 u_{t} \gamma^{\prime}\left(\beta \mathscr{J} * u_{t}\right)+v_{t}\right]^{1 / 2} d W_{t} .\right.
\end{aligned}
$$

Here, $d^{2} V$ is the second Frechet derivative of $V$ from (1.7), i.e.

$$
d^{2} V(u) \zeta=i^{\prime \prime}(u) \zeta-\beta \mathscr{J} * \zeta,
$$

and $W_{t}$ is the $\mathscr{S}^{\prime}$-valued Brownian motion with covariance

$$
E\left\langle\varphi, W_{s}\right\rangle\left\langle\psi, W_{t}\right\rangle=(s \wedge t)\langle\varphi, \psi\rangle
$$

for $\varphi, \psi \in \mathscr{C}^{\infty}(\mathbb{T})$.

To get a better understanding of (1.18), let us suppose that $u_{e}$ is a stable steady state solution of (1.6) and that we are not in a critical situation of a phase transition. This means that $u_{e}$ is a local minimum of $V$ and that the second derivative $d^{2} V$ is a non-degenerate, positive definite operator. Then also $v_{t}$ converges to its stable solution $v_{e}=\gamma^{\prime \prime}\left(\beta \mathscr{J} * u_{e}\right)+\left(\gamma^{\prime}\right)^{2}\left(\beta \mathscr{J} * u_{e}\right)$ such that with $u_{e}=\gamma^{\prime}\left(\beta \mathscr{J} * u_{e}\right),(1.18)$ reduces to

$$
d \zeta_{t}=-\gamma^{\prime \prime}\left(\beta \mathscr{J} * u_{e}\right) \exp \gamma\left(\beta \mathscr{J} * u_{e}\right) d^{2} V\left(u_{e}\right) \zeta_{t} d t+\left[2 \exp \gamma\left(\beta \mathscr{J} * u_{e}\right) \gamma^{\prime \prime}\left(\beta \mathscr{J} * u_{e}\right)\right]^{1 / 2} d W_{t} .
$$

Thus, $\zeta_{t}$ is a generalized Ornstein-Uhlenbeck process and its unique stationary distribution is the Gaussian field with mean zero and covariance

$$
E\left\langle\varphi, \zeta_{t}\right\rangle\left\langle\psi, \zeta_{t}\right\rangle=\left\langle\varphi,\left(d^{2} V\left(u_{e}\right)\right)^{-1} \psi\right\rangle
$$

On the other hand, it is a consequence of (1.10), that the conditional fluctuation fields of $Q^{n}$, restricted to a neighborhood $U\left(u_{e}\right)$ of $u_{e}, Q^{n}\left[d\left(\sigma^{n}-u_{e} / n^{1 / 2}\right) \mid U\left(u_{e}\right)\right]$ converges to the mean zero Gaussian field with covariance (1.22) (see also [9]).

In order to investigate the situation at a critical point of a phase transition, we must specify our assumptions in order to make sure that a phase transition indeed occurs.

First, we assume $\rho$ to be an even probability measure on $\mathbb{R}$ with compact support and that the GHS-inequality hold (cf. [8]), a consequence of which is that for some $K_{0} \geqq 2$,

$$
0=\gamma(0)=\gamma^{\prime}(0), \quad \gamma^{\prime \prime}(0)>0, \quad 0=\gamma^{(3)}(0)=\cdots=\gamma^{\left(2 K_{0}-1\right)}(0), \quad \gamma^{\left(2 K_{0}\right)}(0)<0 .
$$

Again, $\mathscr{J}$ should be sufficiently smooth and symmetric. For a ferromagnetic phase 
transition, we want its Fourier coefficients to satisfy

$$
\hat{\mathscr{J}}(0)-\hat{\mathscr{J}}(p) \geqq \delta_{0}>0 \text { for all } p \in \mathbb{Z}-\{0\} .
$$

From [7] or [2], we know that a phase transition indeed occurs at the critical inverse temperature

$$
\beta_{0}=\left(\gamma^{\prime \prime}(0) \hat{\mathscr{J}}(0)\right)^{-1} \text {. }
$$

Here, the potential $V=V_{\beta_{0}}$ has a unique minimum at $u_{e} \equiv 0$, but $d^{2} V\left(u_{e}\right)$ has a one-dimensional kernel spanned by the constant function 1. Equation (1.24) requires that the remainder of the spectrum is positive, bounded away from zero by $\beta_{0} \delta_{0}$. We define the critical fluctuation process by

$$
\xi_{t}^{n}=n^{1 / 2 K_{0}} \sigma_{t n^{1-1 / K_{0}}}^{n},
$$

where the new time scale $t n^{1-1 / K_{0}}$ compensates the effect of critical slowing down, mentioned above. We decompose $\xi_{t}^{n}$ into its ferromagnetic component $\theta_{t}^{n}=\xi_{t}^{n}(0) \lambda^{n}$, where $\lambda^{n}=n^{-1} \sum_{x \in \mathbb{T}_{n}} \delta_{x}$ is the discrete Haar measure on $n^{-1} \mathbb{Z} / \mathbb{Z}$, and its complement $\eta_{t}^{n}$

$$
\xi_{t}^{n}=\theta_{t}^{n}+\eta_{t}^{n}
$$

Since $d^{2} V(0)$ is not degenerate in the direction of $\eta_{t}^{n}$, the stronger scaling $n^{1 / 2 K_{0}}$, instead of $n^{1 / 2}$ at the non-critical fluctuation, has the effect that the processes $\eta_{t}^{n}$ collapse to the zero process, and the dynamics of $\theta_{t}^{n}$, in which direction $d^{2} V(0)$ is degenerate, has to be expanded to higher order terms of $\theta_{t}^{n}$. For the following result on critical fluctuations, we need in addition some more complicated asumptions on the starting configurations $\xi_{0}^{n}$, for which we refer to Sect. 6, mainly to insure that $\eta_{0}^{n}$ already collapses sufficiently fast.

Theorem 5'. The critical fluctuation process $\xi_{t}^{n}=\theta_{t}^{n}+\eta_{t}^{n}$ converges in law to the one-dimensional process $\xi_{t}=\hat{\theta}_{t}(0) \lambda$ with

$$
d \hat{\theta}_{t}(0)=\gamma^{\left(2 K_{0}\right)}(0)\left[\left(2 K_{0}-1\right) !\left(\gamma^{\prime \prime}(0)\right)^{2 K_{0}-1}\right]^{-1} \hat{\theta}_{t}^{2 K_{0}-1}(0) d t+\left(2 \gamma^{\prime \prime}(0)\right)^{1 / 2} d w_{t},
$$

and with $w_{t}$ as the standard Brownian motion.

The stationary distribution of the process $\hat{\theta}_{t}(0)$ is given by the non-Gaussian distribution

$$
\exp \left[\gamma^{\left(2 K_{0}\right)}(0)\left(2\left(2 K_{0}\right) !\left(\gamma^{\prime \prime}(0)\right)^{2 K_{0}}\right)^{-1} \theta^{2 K_{0}}\right] d \theta / Z_{1},
$$

with normalizing constant $Z_{1}$. Notice that the surviving process $\hat{\theta}_{t}(0)$ in $(1.28)$ depends only on quantities coming from the cumulant generating function $\gamma$ of the single spin distribution $\rho$. It is invariant from the specific interaction function $\mathscr{J}$, except for the implicit assumption that we are indeed at the critical point of the ferromagnetic second-order phase transition. This phenomenon is called universality.

In first approximation, the time evolution of the system is space-homogeneous, since the fluctuation process is concentrated on constant densities: this shows that the ferromagnetic order comes through the fluctuations at the critical temperature.

This kind of result on critical fluctuation processes was first obtained by Dawson 
[5] for a non-geometric model with mean-field interaction, with a one-dimensional kernel of the second derivative of the large deviation potential $V$ at the critical point, this proof is based on a semi-group perturbation theory. Our proofs use martingale decompositions and martingale inequalities, which allow us in Sect. 7 to treat also critical fluctuations at an antiferromagnetic phase transition, where the kernel of $d^{2} V(0)$ has dimension 2. However, we have to strengthen the assumption (1.23) by requiring

$$
\gamma^{(4)}(0)<0 ; \quad \text { i.e. } \quad K_{0}=2,
$$

and instead of (1.24-25), we now have for $\hat{\mathscr{J}}\left(p_{0}\right)=\hat{\mathscr{J}}\left(-p_{0}\right)$,

$$
\begin{aligned}
\hat{\mathcal{J}}\left(p_{0}\right)-\hat{\mathscr{J}}(q) & \geqq \delta_{0}>0 \quad \text { for all } \quad q \in \mathbb{Z} \backslash\left\{ \pm p_{0}\right\}, \\
\beta_{p_{0}} & =\left(\gamma^{\prime \prime}(0) \hat{\mathscr{J}}\left(p_{0}\right)\right)^{-1} .
\end{aligned}
$$

These conditions assure that we are at the critical point of a second-order phase transition to an antiferromagnetic state with frequency $p_{0}$ (cf. [2]). This time, we split the critical fluctuation process $\xi_{t}^{n}=n^{1 / 4} \sigma_{t n^{1 / 2}}^{n}$ into the two-dimensional $p_{0^{-}}$ antiferromagnetic components

$$
\varphi_{t}^{n}=\left[2 \operatorname{Re}\left(\widehat{\zeta_{t}^{n}}\left(p_{0}\right)\right) \cos \left(2 \pi p_{0} x\right)+2 \operatorname{Im}\left(\widehat{\zeta_{t}^{n}}\left(p_{0}\right)\right) \sin \left(2 \pi p_{0} x\right)\right] \lambda^{n}(d x),
$$

and its complement $\psi_{t}^{n}: \xi_{t}^{n}=\varphi_{t}^{n}+\psi_{t}^{n}$.

Here, we again omit the assumptions on the initial configurations.

Theorem 6'. At the critical point of an antiferromagnetic phase transition of frequency $p_{0}$, the critical fluctuation process $\xi_{t}^{n}$ converges in law to the two-dimensional antiferromagnetic process of frequency $p_{0}$

$$
\varphi_{t}(d x)=2\left[\operatorname{Re}\left(\hat{\varphi}_{t}\left(p_{0}\right)\right) \cos \left(2 \pi p_{0} x\right)+\operatorname{Im}\left(\hat{\varphi}_{t}\left(p_{0}\right)\right) \sin \left(2 \pi p_{0} x\right)\right] \lambda(d x),
$$

where $\hat{\varphi}_{t}\left(p_{0}\right) \in \mathbb{C}$ is given by

$$
d \hat{\varphi}_{t}\left(p_{0}\right)=\gamma^{(4)}(0)\left(2 \gamma^{\prime \prime}(0)^{3}\right)^{-1}\left|\varphi_{t}\left(p_{0}\right)\right|^{2} \hat{\varphi}_{t}\left(p_{0}\right) d t+\left(2 \gamma^{\prime \prime}(0)\right)^{1 / 2} d w_{t}^{\mathbb{C}},
$$

with $w_{t}^{\mathbb{C}}$ the complex Brownian motion.

Again, the stationary distribution of $\hat{\varphi}_{t}\left(p_{0}\right) \in \mathbb{C}$ is non-Gaussian:

$$
\exp \left\{\gamma^{(4)}(0)\left(16\left(\gamma^{\prime \prime}(0)\right)^{4}\right)^{-1}|Z|^{4}\right\} d Z / Z_{2}
$$

with normalizing $Z_{2}$. This time, the system at equilibrium behaves in first approximation as a random sine-like wave, with fixed frequency $p_{0}$, but whose phase and magnitude are coupled random processes; here again, the system is brought to an antiferromagnetic order by its fluctuations.

Finally, we calculate in Sect. 8 the limit of the critical fluctuation process at a triple point, where a ferromagnetic and an antiferromagnetic phase transition fall together. This means that for some $p_{0} \neq 0$,

$\hat{\mathscr{J}}(0)=\hat{\mathscr{J}}\left(p_{0}\right)=\hat{\mathscr{J}}\left(-p_{0}\right), \quad \hat{\mathscr{J}}(0)-\hat{\mathscr{J}}(q) \geqq \delta_{0}>0 \quad$ for all $\quad q \in \mathbb{Z} \backslash\left\{0, \pm p_{0}\right\}$

and

$$
\beta_{0}=\left(\gamma^{\prime \prime}(0) \hat{\mathscr{J}}(0)\right)^{-1} .
$$


Now in the infinite particle limit, the critical fluctuation process has the form

$$
\mu_{t}(d x)=\left[\hat{\mu}_{t}(0)+2 \operatorname{Re}\left(\hat{\mu}_{t}\left(p_{0}\right)\right) \cos \left(2 \pi p_{0} x\right)+2 \operatorname{Im}\left(\hat{\mu}_{t}\left(p_{0}\right)\right) \sin \left(2 \pi p_{0} x\right)\right] \lambda(d x),
$$

and $\left(\hat{\mu}_{t}(0), \hat{\mu}_{t}\left(p_{0}\right)\right) \in \mathbb{R} \times \mathbb{C}$ is driven by the coupled stochastic differential equation

$$
\begin{aligned}
d \hat{\mu}_{t}(0) & =\gamma^{(4)}(0)\left(3 !\left(\gamma^{\prime \prime}(0)\right)^{3}\right)^{-1}\left(\hat{\mu}_{t}^{2}(0)+6\left|\hat{\mu}_{t}\left(p_{0}\right)\right|^{2}\right) \hat{\mu}_{t}(0) d t+\left[2 \gamma^{\prime \prime}(0)\right]^{1 / 2} d w_{t}, \\
d \hat{\mu}_{t}\left(p_{0}\right) & =\gamma^{(4)}(0)\left(2\left(\gamma^{\prime \prime}(0)\right)^{3}\right)^{-1}\left(\hat{\mu}_{t}^{2}(0)+\left|\hat{\mu}_{t}\left(p_{0}\right)\right|^{2}\right) \hat{\mu}_{t}\left(p_{0}\right) d t+\left[2 \gamma^{\prime \prime}(0)\right]^{1 / 2} d w_{t}^{\mathbb{C}}
\end{aligned}
$$

with $w_{t}$ and $w_{t}^{\mathbb{C}}$ independent real, respectively complex Brownian motions.

In the appendix, we add a useful proposition on collapsing processes, which is of interest in its own right.

\section{Notations and Main Example}

Let $\mathbb{T}$ be the $d$-dimensional torus $(\mathbb{R} / \mathbb{Z})^{d}$. For any natural number $n \in \mathbb{N}$ we consider the lattice torus $\mathbb{T}_{n}=\left(n^{-1} \mathbb{Z} / \mathbb{Z}\right)^{d}$ with spacing $n^{-1}$, consisting of the $N=n^{d}$ sites $x=\left(k_{1} / n, \ldots, k_{d} / n\right)$, where $k_{j}=0, \ldots, n-1$ for $j=1, \ldots, d$.

To each lattice site $x \in \mathbb{T}_{n}$, we associate a real-valued spin $\sigma(x)$, whose ensemble defines the magnetization field

$$
\sigma^{n}=N^{-1} \sum_{x \in \mathbb{T}_{n}} \sigma(x) \delta_{x} \in \mathscr{M},
$$

with $\delta_{x}$ the Dirac mass at $x$ and $\mathscr{M}=M(\mathbb{T})$ the set of Radon measures on $\mathbb{T}$. We endow $\mathscr{M}$ with the weak-* topology, which makes $\mathscr{M}$ a metrisable space. Let $\mathscr{M}^{n}$ be the set of all measure of the form (2.1), $\mathscr{M}_{b}^{n}=\left\{\sigma^{n} \in \mathscr{M}^{n},|\sigma(x)| \leqq b\right.$ for all $\left.x \in \mathbb{T}_{n}\right\}$, and $\mathscr{M}_{b}=\{\mu \in \mathscr{M},\|\mu\| \leqq b\}$, where $\|\mu\|$ means the total variation of $\mu$. $\mathscr{M}^{n}$ and $\mathscr{M}_{b}^{n}$ are closed subsets of $\mathscr{M}$, respectively $\mathscr{M}_{b}$, and $\mathscr{M}_{b}$ is compact in the weak-* topology.

We assume the single spin distribution $\rho$ to be a probability measure on $\mathbb{R}$ with compact support ${ }^{1}$, say contained in $B=[-b,+b]$. (In Sect. 5, we shall impose further restrictions on $\rho$.) Let

$$
\gamma(u)=\log \int \exp \{m u\} \rho(d m)
$$

be the logarithm of the moment generating function. $\gamma$ is a convex function with $\gamma(0)=0$. Note that

$$
\begin{aligned}
\int m \exp \{m u\} \rho(d m) & =\gamma^{\prime}(u) \exp \gamma(u), \\
\int m^{2} \exp \{m u\} \rho(d m) & =\left[\gamma^{\prime \prime}(u)+\left(\gamma^{\prime}(u)\right)^{2}\right] \exp \gamma(u) .
\end{aligned}
$$

Let $\lambda$ be the Lebesgue measure on $\mathbb{T}$ and

$$
\lambda^{n}=N^{-1} \sum_{x \in \mathbb{T}_{n}} \delta_{x}
$$

\footnotetext{
${ }^{1}$ We could also consider distributions $\rho$ with unbounded support, provided that $\gamma$ is finite on the whole line and some boundedness assumptions on the flipping rates. Then, proving exponential estimates on the tail distribution of the process, we are reduced to the case of bounded $\rho$. For gaussian spins, i.e. when $\rho$ is a Gauss distribution, the assumptions amount to high temperature (one phase situations), since critical temperature corresponds to explosion of the system
} 
its discrete analogue on $\mathbb{T}_{n}$. Finally for $\sigma \in \mathscr{M}, m \in \mathbb{R}$, we define

$$
\left.\sigma\right|_{x} ^{n} m(\cdot)=\sigma\left(\cdot \backslash C_{n}(x)\right)+m / N \delta_{x}(\cdot)
$$

where $C_{n}(x)=\left(x_{1}-1 / 2 n, x_{1}+1 / 2 n\right] \times \cdots \times\left(x_{d}-1 / 2 n, x_{d}+1 / 2 n\right] \subseteq \mathbb{T}$ is the cube in $T$ with centre $x$ and edge length $1 / n$.

Now we define the operators $L^{n}$ on $\mathscr{C}(\mathscr{M})$ by

$$
L^{n} f(\sigma)=\int_{B \times \mathbb{T}}\left[f\left(\left.\sigma\right|_{x} ^{n} m\right)-f(\sigma)\right] N A^{n}(m, x, \sigma) \rho(d m) \lambda^{n}(d x)
$$

with

and

$$
\begin{gathered}
A^{n}(m, x, \sigma)=\exp \left\{G_{0}(x, \sigma)+m G_{1}(x, \sigma)+G_{2}^{n}(m, x, \sigma)\right\}, \\
G_{0}, G_{1} \in \mathscr{C}(\mathbb{T} \times \mathscr{M}),
\end{gathered}
$$

$$
G_{2}^{n} \underset{n \rightarrow \infty}{\longrightarrow} 0
$$

in a sense to be made precise in the following sections.

We set

$$
A(m, x, \sigma)=\exp \left\{G_{0}(x, \sigma)+m G_{1}(x, \sigma)\right\} .
$$

clearly, there exists a unique Markov process $P^{n}$ on the Skorokhod space $\Omega=\mathscr{D}\left(\mathbb{R}^{+}, \mathscr{M}\right)$, the space of right-continuous, $\mathscr{M}$-valued functions with left-hand limits, with $L^{n}$ as its infinitesimal generator, i.e.

$$
f\left(\sigma_{t}\right)-f\left(\sigma_{0}\right)-\int_{(0, t]} L^{n} f\left(\sigma_{s}\right) d s=M_{t}^{n}(f) \text { is a } P^{n} \text {-martingale }
$$

for all $f \in \mathscr{C}(\mathscr{M})$. This martingale can be written in the integral form

$$
M_{t}^{n}(f)=\int_{(0, t]} \int_{B \times \pi}\left[f\left(\sigma_{s-} \mid{ }_{x}^{n} m\right)-f\left(\sigma_{s-}\right)\right] \tilde{\Lambda}^{n}(d m, d x, d s)
$$

where for $\sigma \in \Omega$

$$
\tilde{\Lambda}^{n}(d m, d x, d s)(\sigma)=\Lambda^{n}(d m, d x, d s)(\sigma)-N A^{n}\left(m, x, \sigma_{s}\right) \rho(d m) \lambda^{n}(d x) d s
$$

with a pure point process $\Lambda^{n}(d m, d x, d s)(\sigma)$. The corresponding increasing process (see [14], II.3.9) is

$$
\left\langle M^{n}(f), M^{n}(f)\right\rangle_{t}=\int_{0}^{t} \int_{B \times \mathbb{T}}\left[f\left(\left.\sigma_{s}\right|_{x} ^{n} m\right)-f\left(\sigma_{s}\right)\right]^{2} N A^{n}\left(m, x, \sigma_{s}\right) \rho(d m) \lambda^{n}(d x) d s .
$$

Example. The general $q$-body long-range interaction between the spins of a magnetic field has the internal energy

$$
H(\sigma)=-\sum_{j=1}^{q} 1 / j ! \int_{J^{j}} \mathscr{J}_{j}\left(x_{1}, \ldots, x_{j}\right) \sigma\left(d x_{1}\right) \cdots \sigma\left(d x_{j}\right)=-\sum_{j=1}^{q} 1 / j !\left\langle\mathscr{J}_{j}, \sigma^{\otimes j}\right\rangle,
$$

where $\mathscr{J}_{j} \in \mathscr{C}\left(\mathbb{T}^{j}\right)$. Its Frechet derivative is

$$
\nabla H(\sigma)(x)=-\sum_{j=1}^{q} 1 / j ! \sum_{i=1}^{j}\left\langle\mathscr{J}_{j}, \sigma^{\otimes i-1} \otimes \delta_{x} \otimes \sigma^{\otimes j-1}\right\rangle \in \mathscr{C}(\mathbb{T}) .
$$


Now, let $G_{0}$ be any continuous function on $\mathbb{T} \times \mathscr{M}$, with

and

$$
\sup _{x \in \mathbb{J}_{n}, \sigma \in \mathscr{M}_{b}^{n}}\left|G_{0}(x, \sigma)-G_{0}\left(x,\left.\sigma\right|_{x} ^{n} 0\right)\right|=\mathcal{O}\left(N^{-1}\right),
$$

$$
G_{1}(x, \sigma)=-\beta \nabla H(\sigma)(x),
$$

where $\beta>0$ is the inverse temperature. We set

$$
\begin{aligned}
G_{2}^{n}(m, x, \sigma)= & G_{0}\left(x,\left.\sigma\right|_{x} ^{n} 0\right)-G_{0}(x, \sigma)+\beta\left\{N H(\sigma)-N H\left(\left.\sigma\right|_{x} ^{n} 0\right)\right. \\
& \left.-\sigma(x) \nabla H\left(\left.\sigma\right|_{x} ^{n} 0\right)+m\left(\nabla H(\sigma)-\nabla H\left(\left.\sigma\right|_{x} ^{n} 0\right)\right)\right\} .
\end{aligned}
$$

By (2.15-17), it is easy to check that

$$
\sup \left\{\left|G_{2}^{n}(m, x, \sigma)\right| ; m \in B, x \in \mathbb{T}_{n}, \sigma \in \mathscr{M}_{b}^{n}\right\}=\mathcal{O}\left(N^{-1}\right) .
$$

The detailed balanced condition (see [27]) shows that the unique invariant probability distribution for the process $P^{n}$ with infinitesimal generator $L^{n}$, given by $(2.7-8)$, is the Gibbs state

$$
Q^{n}\left(d \sigma^{n}\right)=\exp \left\{-\beta N H\left(\sigma^{n}\right)\right\} \prod_{x \in \mathbb{T}_{n}} \rho(d \sigma(x)) / Z^{n}
$$

with $\sigma^{n}$ from (2.1) and $Z^{n}$ as normalizing constant. The thermodynamic limit of (2.21) has been investigated in [7].

\section{Asymptotic Dynamics of the Magnetization}

Besides (2.9-10), we assume that

$G_{0}$ and $G_{1}$ are Lipschitz-continuous in $\sigma \in \mathscr{M}_{b}$ in the total variation norm, and that

$$
\sup \left\{\left|G_{2}^{n}(m, x, \sigma)\right| ; m \in B, x \in \mathbb{T}_{n}, \sigma \in \mathscr{M}_{b}^{n}\right\}=o(1)
$$

Set

$$
\left(\sigma^{n}\right)^{2}(d x)=N^{-1} \sum_{y \in \mathbb{T}_{n}} \sigma^{2}(y) \delta_{y}(d x)
$$

\section{Theorem 1}

(i) Let $\sigma_{0}^{n} \in \mathscr{M}_{b}^{n}$ converge in law to $u_{0} \lambda$, i.e.

$$
u_{0} \in L_{b}^{\infty}=\left\{u \in L^{\infty},\|u\|_{\infty} \leqq b\right\} .
$$

Then the process $\left(\sigma_{t}^{n}\right)_{t \leqq T}$ converges in law to $\left(u_{t} \lambda\right)_{t \leqq T}$, where $u_{t} \in L_{b}^{\infty}$ is the unique solution of the mean-field evolution equation ${ }^{2}$,

$$
d / d t u_{t}=G\left(u_{t}\right)
$$

starting at $u_{0}$, and

$$
G(u)(x)=\exp \left\{G_{0}(x, u)+\gamma\left(G_{1}(x, u)\right)\right\}\left[\gamma^{\prime}\left(G_{1}(x, u)\right)-u(x)\right] .
$$

\footnotetext{
${ }^{2}$ In fact, this convergence holds (in probability) with an exponential rate; refer to [1] for the case of Ising spins
} 
(ii) Moreover, let $\left(\sigma_{0}^{n}\right)^{2}$ converge in law to some $v_{0} \lambda$,

$$
v_{0} \in L_{\left[0, b^{2}\right]}^{\infty}=\left\{\alpha \in L^{\infty} ; 0 \leqq \operatorname{ess} \inf \alpha \leqq \text { ess sup } \alpha \leqq b^{2}\right\} .
$$

Then, $\left(\sigma_{t}^{n}\right)^{2}$ converges in law to $v_{t} \lambda$, where $v_{t} \in L_{\left[0, b^{2}\right]}^{\infty}$ is the unique solution of

starting at $v_{0}$, with

$$
d / d t v_{t}=F\left(u_{t}, v_{t}\right)
$$

$$
F(u, v)(x)=\exp \left\{G_{0}(x, u)+\gamma\left[G_{1}(x, u)\right]\right\}\left\{\gamma^{\prime \prime}\left[G_{1}(x, u)\right]+\left(\gamma^{\prime}\left[G_{1}(x, u)\right]\right)^{2}-v(x)\right\} .
$$

Proof. The Lipschitz properties of $G_{0}$ and $G_{1}$ imply that (3.4) and (3.6) have unique solutions. Since $\gamma^{\prime}(y) \in(-b,+b)$ and $\gamma^{\prime \prime}(y)+\left(\gamma^{\prime}\right)^{2}(y) \in\left(0, b^{2}\right)$ for all $b \in \mathbb{R}$ the solutions $u_{t}, v_{t}$, satisfy $-b \leqq u_{t} \leqq b, 0 \leqq v_{t} \leqq b^{2}$. In order to show the tightness of $\left(\sigma_{t}^{n}\right)_{t=T}$, notice that $\sigma_{0}^{n} \in \mathscr{M}_{b}^{n}$ implies $\sigma_{t}^{n} \in \mathscr{M}_{b}^{n} \leqq \mathscr{M}_{b}$ for all $t \geqq 0, P^{n}$ a.e., and that $\mathscr{M}_{b}$ is compact in its weak-* topology. It suffices therefore to show uniform continuity in the following form:

For any $g \in \mathscr{C}(\mathbb{T}), \eta, \varepsilon>0$, there exists $n_{0} \in \mathbb{N}$ and $\delta>0$ such that

$$
\sup _{n \geqq n_{0} 0 \leqq \tau_{1} \leqq \tau_{2} \leqq\left(\tau_{1}+\delta\right) \wedge T} P^{n}\left\{\left|\left\langle g, \sigma_{\tau_{2}}^{n}\right\rangle-\left\langle g, \sigma_{\tau_{n}}^{n}\right\rangle\right|>\eta\right\} \leqq \varepsilon
$$

where $\tau_{1}, \tau_{2}$ are stopping times (cf. [16], I.3.4). From (2.7-13), we get

$$
\begin{aligned}
\left\langle g, \sigma_{\tau_{2}}^{n}\right\rangle-\left\langle g, \sigma_{\tau_{1}}^{n}\right\rangle= & N^{-1} \int_{\left(\tau_{1}, \tau_{2}\right]_{B \times \mathbb{T}}} \int_{B \times} g(x)\left(m-\sigma_{s-}^{n}(x)\right) \tilde{\Lambda}^{n}(d m, d x, d s) \\
& +\int_{\tau_{1}}^{\tau_{2}} \int_{B \times \mathbb{J}} g(x)\left(m-\sigma_{s}^{n}(x)\right) A^{n}\left(m, x, \sigma_{s}^{n}\right) \rho(d m) \lambda^{n}(d x) d s
\end{aligned}
$$

with the last term being in absolute value less than $2 b\|g\|_{\infty}\|A+1\|_{\infty} \cdot \delta$ for $n$ sufficiently large, using (3.2). Therefore, by (2.14)

$$
\begin{aligned}
& P^{n}\left\{\left|\left\langle g, \sigma_{\tau_{2}}^{n}\right\rangle-\left\langle g, \sigma_{\tau_{1}}^{n}\right\rangle\right|>\eta\right\} \leqq \eta^{-2} E^{n}\left(\left(\left\langle g, \sigma_{\tau_{2}}^{n}\right\rangle-\left\langle g, \sigma_{\tau_{2}}^{n}\right\rangle\right)^{2}\right) \\
& \quad \leqq 8 b^{2}\|g\|_{\infty}^{2}\|A+1\|_{\infty}^{2} \cdot \delta^{2} / \eta^{2}+N^{-1} 8 b^{2}\|g\|_{\infty}^{2}\|A+1\|_{\infty} \cdot \delta / \eta^{2},
\end{aligned}
$$

which is less than $\varepsilon$ for all $n \in \mathbb{N}$, if $\delta$ is sufficiently small. Furthermore, the jump sizes go to zero uniformly, so any limit law is concentrated on continuous paths.

The tightness of the processes $\left(\sigma_{t}^{n}\right)_{t \leqq T}^{2}$ is shown similarly. As in (3.10), we get by Doob's inequality

$$
P^{n}\left\{\sup _{t \leqq T}\left|N^{-1} \int_{0}^{t} \int_{B \times \mathbb{T}} g(x)\left(m-\sigma_{s-}^{n}(x)\right) \tilde{\Lambda}^{n}(d m, d x, d s)\right|>\eta N^{-1 / 3}\right\}=\mathcal{O}\left(N^{-1 / 3}\right) .
$$

Hence, outside of a set of very small $P^{n}$-probability, we have

$$
\begin{gathered}
\left\langle g, \sigma_{t}^{n}\right\rangle=\left\langle g, \sigma_{0}^{n}\right\rangle+\int_{0}^{t} \int_{B \times \pi} g(x)\left(m-\sigma_{s}^{n}(x)\right) A^{n}\left(m, x, \sigma_{s}^{n}\right) \rho(m) \lambda^{n}(d x) d s+o(1) \\
=\left\langle g, \sigma_{0}^{n}\right\rangle+\int_{0}^{t} \int_{T} g(x) \exp \left\{G_{0}\left(x, \sigma_{s}^{n}\right)+\gamma\left(G_{1}\left(x, \sigma_{s}^{n}\right)\right)\right\} \\
{\left[\gamma^{\prime}\left(G_{1}\left(x, \sigma_{s}^{n}\right)\right) \lambda^{n}(d x)-\sigma_{s}^{n}(d x)\right] d s+o(1) .}
\end{gathered}
$$


But the maps $\mu \mapsto G_{i}(., \mu) \in \mathscr{C}(\mathbb{T}), i=1,2$, are continuous on the (compact) set $\mathscr{M}_{b}$; then, from Ascoli's theorem, their range is a uniformly equicontinuous family of $\mathscr{C}(\mathbb{T})$, and the Riemann sum $\lambda^{n}$ in the last term converges uniformly to the $\lambda$-integral. Combining this with (3.5), we derive that with large probability

$$
\left\langle g, \sigma_{t}^{n}\right\rangle=\left\langle g, \sigma_{0}^{n}\right\rangle+\int_{0}^{t}\left\langle g, G\left(\sigma_{s}^{n}\right)\right\rangle d s+o(1),
$$

and so any limit process of $\left(\sigma_{t}^{n}\right)_{t \leqq T}$ must be concentrated on a solution of (3.4), which is unique however. In the case of $\left(\sigma^{n}\right)^{2}$, we obtain

$$
\begin{aligned}
\left\langle g,\left(\sigma_{t}^{n}\right)^{2}\right\rangle= & \left\langle g,\left(\sigma_{0}^{n}\right)^{2}\right\rangle+\int_{0}^{t} \int_{B \times \mathbb{T}} g(x)\left(m^{2}-\left(\sigma_{s}^{n}(x)\right)^{2}\right) A^{n}\left(m, x, \sigma_{s}^{n}\right) \rho(d m) \lambda^{n}(d x) d s+o(1) \\
= & \left\langle g,\left(\sigma_{0}^{n}\right)^{2}\right\rangle+\int_{0}^{t} \int_{\mathbb{J}} g(x) \exp \left\{G_{0}\left(x, \sigma_{s}^{n}\right)+\gamma\left(G_{1}\left(x, \sigma_{s}^{n}\right)\right)\right\} \\
& \cdot\left[\left(\gamma^{\prime \prime}\left(G_{1}\left(x, \sigma_{s}^{n}\right)\right)+\left(\gamma^{\prime}\right)^{2}\left(G_{1}\left(x, \sigma_{s}^{n}\right)\right)\right) \lambda(d x)-\left(\sigma_{s}^{n}\right)^{2}(d x)\right] d s+o(1) \\
= & \left\langle g,\left(\sigma_{0}^{n}\right)^{2}\right\rangle+\int_{0}^{t}\left\langle g, F\left(\sigma_{s}^{n},\left(\sigma_{s}^{n}\right)^{2}\right)\right\rangle d s+o(1)
\end{aligned}
$$

This completes the proof of the theorem.

Remark. Point ii) of Theorem 1 follows from Theorem 2, which requires stronger assumptions.

\section{Asymptotic Dynamics of the Densities and Propagation of Chaos}

To a magnetization field $\sigma^{n} \in \mathscr{M}_{b}^{n}$, we associate the empirical magnetization density

$$
\pi^{n}=N^{-1} \sum_{x \in \mathbb{T}_{n}} \delta_{\left(\sigma^{n}(x), x\right)} \in \mathscr{P}(B \times \mathbb{T})
$$

where $\mathscr{P}(B \times \mathbb{T})$ denotes the set of all probability measures on $B \times \mathbb{T} . \mathscr{P}(B \times \mathbb{T})$ is compact in the weak-* topology.

We first show that the density process $\pi^{n}$ converges to a deterministic density, governed by the asymptotic magnetization process:

Theorem 2. Assume (3.1-2) and that $\pi_{0}^{n}$ converge in law to $h_{0}(m, x) \rho(d m) \lambda(d x) \in$ $\mathscr{P}(B \times \mathbb{T}), h_{0} \in L^{\infty}(B \times \mathbb{T})$. Then the empirical density process $\pi_{t}^{n}$ converges in law to $h_{t}(m, x) \rho(d m) \lambda(d x)$, where the density $h_{t} \in L^{\infty}(B \times \mathbb{T})$ is the solution

$$
d / d t h_{t}(m, x)=\exp \left\{G_{0}\left(x, u_{t}\right)+m G_{1}\left(x, u_{t}\right)\right\}-h_{t}(m, x) \exp \left\{G_{0}\left(x, u_{t}\right)+\gamma\left(G_{1}\left(x, u_{t}\right)\right)\right\},
$$

starting at $h_{0}$; and where $u_{t}(x)=\int_{B} m h_{t}(m, x) \rho(d m)$ is the solution of $(3.4)$ with $u_{0}(x)=$ $\int_{B} m h_{0}(m, x) \rho(d m)$.

Since by $(2.9-10)$

$$
\sup \left\{\exp \left[m G_{1}(x, \sigma)-\gamma\left(G_{1}(x, \sigma)\right)\right] ; m \in B, x \in \mathbb{T}, \sigma \in \mathscr{M}_{b}\right\}=C<\infty,
$$

$0 \leqq h_{t}(m, x) \leqq C$, if this property holds for $h_{0}$. Therefore $h_{t} \in L^{\infty}(B \times \mathbb{T})$ for all $t$. 
Moreover

$$
\int h_{t}(m, x) \rho(d m)=1 \text {. }
$$

Proof. Since (4.2) is linear in $h$, it has a unique solution in $L^{\infty}(B \times \mathbb{T})$ satisfying (4.4). Let $g \in \mathscr{C}(B \times \mathbb{T})$. Then

$$
\begin{aligned}
\left\langle g, \pi_{t}^{n}\right\rangle= & \left\langle g, \pi_{0}^{n}\right\rangle+\int_{0}^{t} \int_{B \times \mathbb{T}}\left[g(m, x)-g\left(\sigma_{s}^{n}(x), x\right)\right] A^{n}\left(m, x, \sigma_{s}^{n}\right) \rho(d m) \lambda^{n}(d x) d s \\
& +\int_{0}^{t} \int_{B \times \mathbb{T}} N^{-1}\left[g(m, x)-g\left(\sigma_{s-}^{n}(x), x\right)\right] \tilde{\Lambda}^{n}(d m, d x, d s) .
\end{aligned}
$$

The uniform continuity can now be shown in the same way as is $(3.8-10)$. By the compactness of $\mathscr{P}(B \times \mathbb{T})$, the sequence of processes $\pi_{t}^{n}$ is therefore tight.

Doob's inequality implies again

$$
P^{n}\left\{\sup _{t \leqq T}\left|\int_{0}^{t} \int_{B \times \mathbb{T}} N^{-1}\left[g(m, x)-g\left(\sigma_{s-}^{n}(x), x\right)\right] \tilde{\Lambda}^{n}(d m, d x, d s)\right|>\eta N^{-1 / 3}\right\}=\mathcal{O}\left(N^{-1 / 3}\right)
$$

which gives outside a set of uniformly small probability

$$
\begin{aligned}
\left\langle g, \pi_{t}^{n}\right\rangle= & \left\langle g, \pi_{0}^{n}\right\rangle+\int_{0}^{t}\left[\int_{B \times \mathbb{T}} \exp \left\{G_{0}\left(x, \sigma_{s}^{n}\right)+m G_{1}\left(x, \sigma_{s}^{n}\right)\right\} g(m, x) \rho(d m) \lambda^{n}(d x)\right. \\
& \left.-\int_{B \times \mathbb{T}} \exp \left\{G_{0}\left(x, \sigma_{s}^{n}\right)+\gamma\left(G_{1}\left(x, \sigma_{s}^{n}\right)\right)\right\} g(m, x) \pi_{s}^{n}(d m, d x)\right] d s+o(1) \\
= & \left\langle g, \pi_{0}^{n}\right\rangle+\int_{0}^{t}\left[\int_{B \times \mathbb{J}} \exp \left\{G_{0}\left(x, u_{s}\right)+m G_{1}\left(x, u_{s}\right)\right\} g(m, x) \rho(d m) \lambda(d x)\right. \\
& \left.-\int_{B \times \mathbb{T}} \exp \left\{G_{0}\left(x, u_{s}\right)+\gamma\left(G_{1}\left(x, u_{s}\right)\right)\right\} g(m, x) \pi_{s}^{n}(d m, d x)\right] d s+o(1) \\
& +\mathcal{O}\left(\sup _{s \leqq T} \sup _{x \in \mathbb{T}}\left(\left|G_{0}\left(x, u_{s}\right)-G_{0}\left(x, \sigma_{s}^{n}\right)\right|+\left|G_{1}\left(x, u_{s}\right)-G_{1}\left(x, \sigma_{s}^{n}\right)\right|\right)\right) .
\end{aligned}
$$

Since by Theorem 1, the last term converges to zero, uniformly in probability, we find that any limit $\pi_{s}$ of the processes $\pi_{s}^{n}$ satisfies the following equation, which is deterministic except for $\pi_{0}$ :

$$
\begin{aligned}
\left\langle g, \pi_{t}\right\rangle= & \left\langle g, \pi_{0}\right\rangle+\int_{0}^{t}\left[\int_{B \times T} \exp \left\{G_{0}\left(x, u_{s}\right)+m G_{1}\left(x, u_{s}\right)\right\} g(m, x) \rho(d m) \lambda(d x)\right. \\
& \left.-\int_{B \times \mathbb{T}} \exp \left\{G_{0}\left(x, u_{s}\right)+\gamma\left(G_{1}\left(x, u_{s}\right)\right)\right\} g(m, x) \pi_{s}(d m, d x)\right] d s .
\end{aligned}
$$

But the solution of (4.8) is unique, and if $\pi_{0}=h_{0} d \rho d \lambda$, then also $\pi_{t}$ has a density $h_{t}(m, x)$ with respect to $d \rho d \lambda$, and $h_{t}$ is the solution of (4.2). This completes the proof.

Notice that if $h_{0} \in \mathscr{C}(B \times \mathbb{T})$, then $h_{t} \in \mathscr{C}(B \times \mathbb{T})$ for all $t>0$. Since the right-hand side of (4.2) depends only on the single site $x$, it is obvious that results of the type 
of "propagation of chaos" should hold. In fact, we shall derive two versions of propagations of chaos. The first one will be at the level of empirical measures. In analogy to the weak-* topology on $\mathscr{P}(B \times \mathbb{T})$, used in Theorem 2 , we shall obtain only a weak version at this level.

The second result is the usual "propagation of chaos" for the random spin variables $\sigma_{t}^{n}(x)$. It says that, if the spins at distinct sites are independently distributed at $t=0$, then in the limit $n \rightarrow \infty$, they continue to behave independently at any time $t>0$ according to a distribution which satisfies (4.2), i.e. they constitute a sample of the empirical density. Of course, this is not true for finite $n$, where the spins are dependent. We shall see that this is a consequence of the first version, yielding here an original proof of the standard result.

Let $x_{1}, \ldots, x_{K}$ be distinct sites in $\pi$.

Let $\varepsilon_{n}$ be a sequence of positive numbers with

$$
\varepsilon_{n} \downarrow 0 \text { and } N^{-1} \varepsilon_{n}^{-2 d} \rightarrow 0 \text { as } n \rightarrow \infty .
$$

We define

$$
\bar{\pi}_{t}^{n}=\prod_{i=1}^{K} \bar{\pi}_{t}^{n}\left(x_{i}\right)=\prod_{i=1}^{K}\left[\left(N \varepsilon_{n}^{d}\right)^{-1} \sum_{y_{i} \in C_{\varepsilon_{n}}\left(x_{i}\right) \cap \pi_{n}} \delta_{\sigma_{t}^{n}\left(y_{i}\right)}\right]
$$

as a positive measure on $B^{K}$, where $C_{\varepsilon_{n}}\left(x_{i}\right)$ are the cubes with center $x_{i}$ and edge length $\varepsilon_{n}$.

Theorem 3. Assume (3.1-2), that $\sigma_{0}^{n}$ converges in law to $u_{0} \lambda$, and that $\bar{\pi}_{0}^{n}$ converges in law to $\prod_{i=1}^{K} h_{0}\left(m_{i}, x_{i}\right) \rho\left(d m_{i}\right)$. Then, for $t>0, \bar{\pi}_{t}^{n}$ converges in law to $\prod_{i=1}^{K} h_{t}\left(m_{i}, x_{i}\right) \rho\left(d m_{i}\right)$ with $h_{t}$ satisfying (4.2).

Proof. First, notice that it is enough to prove the theorem for $K=1$, since $\bar{\pi}_{t}^{n}=\prod_{i} \bar{\pi}_{t}^{n}\left(x_{i}\right)$, and since $h\left(., x_{i}\right) d \rho$ is deterministic. Now, for $g \in \mathscr{C}(B)$,

where

$$
\begin{aligned}
\left\langle g, \bar{\pi}_{t}^{n}(x)\right\rangle= & \left\langle g, \bar{\pi}_{0}^{n}(x)\right\rangle+\int_{0}^{t} \int_{B \times \mathbb{T}} \mathbb{1}_{C_{\varepsilon_{n}}(x)}(y) \varepsilon_{n}^{-d}(g(m) \\
& \left.-g\left(\sigma_{s}^{n}(y)\right)\right) A^{n}\left(m, y, \sigma_{s}^{n}\right) \rho(d m) \lambda^{n}(d y) d s+M_{t}^{n},
\end{aligned}
$$

$$
M_{t}^{n}=\int_{0}^{t} \int_{B \times \mathbb{T}} \mathbb{1}_{C_{\varepsilon_{n}}(x)}(y)\left(N \varepsilon_{n}^{d}\right)^{-1}\left(g(m)-g\left(\sigma_{s^{-}}^{n}(y)\right) \tilde{\Lambda}^{n}(d m, d y, d s)\right.
$$

implies $E^{n}\left(\left(M_{t}^{n}\right)^{2}\right)=\mathcal{O}\left(N^{-1} \varepsilon_{n}^{-2 d}\right) \underset{n \rightarrow \infty}{\longrightarrow} 0$ by (4.9).

By the same argument as in the proof of the last theorem, we see that $\bar{\pi}_{t}^{n}(x)$ converges in law to a positive measure $\bar{\pi}_{t}$ on $B$, which satisfies

$$
\begin{aligned}
\left\langle g, \bar{\pi}_{t}(x)\right\rangle= & \left\langle g, \bar{\pi}_{0}(x)\right\rangle+\int_{0}^{t}\left(\int_{B} g(m) \exp \left(G_{0}+m G_{1}\right)\left(x, u_{s}\right) \rho(d m)\right. \\
& \left.\cdot\left\langle g, \bar{\pi}_{s}(x)\right\rangle \exp \left\{G_{0}+\gamma\left(G_{1}\right)\right\}\left(x, u_{s}\right)\right) d s .
\end{aligned}
$$


Equation (4.13) is linear in $\bar{\pi}$, and therefore, has a unique solution, which is $\bar{\pi}_{t}(x)=h_{t}(., x) d \rho$ by $(4.2)$ and the initial condition $\bar{\pi}_{0}(x)=h_{0}(., x) d \rho$.

As a consequence of the last result, we get the propagation of chaos for the random variables $\sigma_{t}^{n}(x)$ : corresponding to the distinct $x_{1}, \ldots, x_{k} \in \mathbb{T}$, let $x_{i}^{n}$ be sequences with

$$
x_{i}^{n} \in \mathbb{T}_{n} \quad \text { and } \quad \lim x_{i}^{n}=x_{i} \text { for } \quad i=1, \ldots, K .
$$

Theorem 3 bis. Besides (3.1-2), (4.14), Assume that $\sigma_{0}^{n}$ converges in law to $u_{0} \lambda$ and that the distribution of $\left(\sigma_{0}^{n}\left(x_{1}^{n}\right), \ldots, \sigma_{0}^{n}\left(x_{k}^{n}\right)\right)$ converges to $\prod_{i=1}^{K} h_{0}\left(., x_{i}\right) d \rho$ as $n \rightarrow \infty$. Then, for $t>0$, the distribution of $\left(\sigma_{t}^{n}\left(x_{1}^{n}\right), \ldots, \sigma_{t}^{n}\left(x_{k}^{n}\right)\right)$ converges to $\prod_{i=1}^{K} h_{t}\left(., x_{i}\right) d \rho$ with $h_{t}\left(., x_{i}\right)$ from (4.2).

Proof. Without loss of generality, we may add the assumption that $\prod_{i} \bar{\pi}_{0}^{n}\left(x_{i}\right)$ converges in law to $\prod_{i=1}^{K} h_{0}\left(., x_{i}\right) d \rho$ : indeed, this assumption may be achieved via the change in the initial distribution of particles in proportion $\mathcal{O}\left(\varepsilon_{n}^{d}\right)$, then being without any influence on the asymptotic distribution of $\left(\sigma_{t}^{n}\left(x_{1}^{n}\right), \ldots, \sigma_{t}^{n}\left(x_{K}^{n}\right)\right)$.

First, we regard the case $K=1$. Let $g \in \mathscr{C}(B)$.

Using (4.11) and a similar expression for $g\left(\sigma_{t}^{n}\left(x_{1}^{n}\right)\right)$, it is easy to get the following inequality:

$$
\begin{aligned}
& \left|E^{n} g\left(\sigma_{t}^{n}\left(x_{1}^{n}\right)\right)-E^{n}\left\langle g, \bar{\pi}_{t}^{n}\left(x_{1}\right)\right\rangle\right| \leqq\left|E^{n} g\left(\sigma_{0}^{n}\left(x_{1}^{n}\right)\right)-E^{n}\left\langle g, \bar{\pi}_{0}^{n}\left(x_{1}\right)\right\rangle\right| \\
& \quad+\int_{0}^{t}\left(2\|g\|_{\infty} \sup _{y \in C_{\varepsilon_{n}}\left(x_{1}\right)}\left\|A^{n}\left(., x_{1}^{n}, . .\right)-A^{n}(., y, . .)\right\|_{\infty}\right. \\
& \left.\quad+\left\|A^{n}\right\|_{\infty}\left|E^{n} g\left(\sigma_{s}^{n}\left(x_{1}^{n}\right)\right)-E^{n}\left\langle g, \bar{\pi}_{s}^{n}\left(x_{1}\right)\right\rangle\right|\right) d s .
\end{aligned}
$$

Hence, Gronwall's lemma together with the assumptions on the initial distributions and Theorem 3 implies

$$
\lim _{n} E^{n} g\left(\sigma_{t}^{n}\left(x_{1}^{n}\right)\right)=\lim _{n} E^{n}\left\langle g, \bar{\pi}_{t}^{n}\left(x_{1}\right)\right\rangle=\int g(m) h_{t}\left(m, x_{1}\right) \rho(d m) .
$$

For the general case, we take $g_{1}, \ldots, g_{K} \in \mathscr{C}(B)$ and $n$ so large that $C_{\varepsilon_{n}}\left(x_{1}\right), \ldots, C_{\varepsilon_{n}}\left(x_{K}\right)$ are all disjoint. Similar to (4.15), we get

$$
\begin{aligned}
& \left|E^{n} \prod_{j=1}^{K} g_{j}\left(\sigma_{t}^{n}\left(x_{j}^{n}\right)\right)-E^{n} \prod_{j=1}^{K}\left\langle g_{j}, \bar{\pi}_{t}^{n}\left(x_{j}\right)\right\rangle\right| \\
& \leqq\left|E^{n} \prod_{j=1}^{K} g_{j}\left(\sigma_{0}^{n}\left(x_{j}^{n}\right)\right)-E^{n} \prod_{j=1}^{K}\left\langle g_{j}, \bar{\pi}_{0}^{n}\left(x_{j}\right)\right\rangle\right| \\
& +\sum_{j=1}^{K} \int_{0}^{t}\left[2 \prod_{i=1}^{K}\left\|g_{i}\right\|_{\infty} \sup _{y \in C_{\varepsilon_{n}}\left(x_{j}\right)}\left\|A^{n}\left(., x_{j}^{n}, . .\right)-A^{n}(., y, . .)\right\|_{\infty}\right. \\
& +\left\|g_{i}\right\|_{\infty}\left\|A^{n}\right\|_{\infty}\left|E^{n} \prod_{i \neq j} g_{i}\left(\sigma_{s}^{n}\left(x_{i}^{n}\right)\right)-E^{n} \prod_{i \neq j}\left\langle g_{i}, \bar{\pi}_{s}^{n}\left(x_{i}\right)\right\rangle\right| \\
& \left.+\left\|A^{n}\right\|_{\infty}\left|E^{n} \prod_{i} g_{i}\left(\sigma_{s}^{n}\left(x_{i}^{n}\right)\right)-E^{n} \prod_{i}\left\langle g_{i}, \bar{\pi}_{s}^{n}\left(x_{i}\right)\right\rangle\right|\right] d s .
\end{aligned}
$$


By an induction hypothesis, the second integrand goes to zero uniformly in $s$, and we conclude by the same argument with Gronwall's lemma as above, that

$$
\lim _{n} E^{n} \prod_{j=1}^{K} g_{j}\left(\sigma_{t}^{n}\left(x_{j}^{n}\right)\right)=\lim _{n} E^{n} \prod_{j=1}^{K}\left\langle g_{j}, \bar{\pi}_{j}^{n}\left(x_{j}\right)\right\rangle=\prod_{j=1}^{K} \int g_{j}\left(m_{j}\right) h_{t}\left(m_{j}, x_{j}\right) \rho\left(d m_{j}\right),
$$

which proves the theorem.

\section{Non-Critical Fluctuations}

For $r \in \mathbb{R}$, we introduce the Sobolev space

with

$$
H_{r}=\left\{\mu \in \mathscr{C}^{\infty}(\mathbb{T})^{\prime} ;\|\mu\|_{r}<+\infty\right\}
$$

$$
\|\mu\|_{r}^{2}=\sum_{p \in \mathbb{Z}^{d}}\left(1+|p|^{2}\right)^{r}|\hat{\mu}(p)|^{2}
$$

where $\hat{\mu}(p)$ are the Fourier coefficients of the distribution $\mu \in \mathscr{C}^{\infty}(\mathbb{T})^{\prime}$

$$
\hat{\mu}(p)=\langle\exp (2 \pi i p .), \mu\rangle, \quad p \in \mathbb{Z}^{d} .
$$

Here, the brackets $\langle.,$.$\rangle denote the duality pairing. H_{r}$ is a Hilbert space with scalar product

$$
\langle\mu, v\rangle_{r}=\sum_{p \in \mathbb{Z}^{d}}\left(1+|p|^{2}\right)^{r} \hat{\mu}(p) \overline{\hat{v}(p)} \in \mathbb{R}
$$

and $H_{r}, H_{-r}$ are then dual spaces. Obviously, for $r_{2} \geqq r_{1} \geqq 0$,

$$
\mathscr{C}^{\infty}(\mathbb{T})=H_{\infty}=\bigcap_{r^{\prime}} H_{r^{\prime}} \subseteq H_{r_{2}} \subseteq H_{r_{1}} \subseteq H_{0}=L^{2} \subseteq H_{-r_{1}} \subseteq H_{-r_{2}} \subseteq H_{-\infty}=\mathscr{C}^{\infty}(\mathbb{T})^{\prime},
$$

and the embedding $H_{r} \subseteq H_{s}$ for any $r \geqq s$ is Hilbert-Schmidt, whenever $r-s>d / 2$, due to the fact that

$$
C_{-r}=\sum_{p \in \mathbb{Z}^{d}}\left(1+|p|^{2}\right)^{-r}<\infty \quad \text { if and only if } r>d / 2 .
$$

In particular, $\left\|\delta_{x}\right\|_{-r}^{2}=\sum_{p}\left(1+|p|^{2}\right)^{-r}=C_{-r}$, so that

$$
\mathscr{M}_{b}^{n} \subseteq\left\{\mu \in H_{-r},\|\mu\|_{-r} \leqq C_{-r}^{1 / 2} b\right\} \quad \text { for } \quad r>d / 2
$$

and

$$
\begin{aligned}
\left\|\lambda-\lambda^{n}\right\|_{-r}^{2} & =\sum_{p \in \mathbb{Z}^{d}}\left(1+|p|^{2}\right)^{-r}\left|N^{-1} \sum_{x \in \mathbb{N}_{n}} \exp (2 \pi i p x)-\delta_{0}\{p\}\right|^{2} \\
& =\sum_{\substack{p \in(n \mathbb{Z})^{d} \\
p \neq 0}}\left(1+|p|^{2}\right)^{-r} \leqq C_{-r}^{\prime} n^{-2 r},
\end{aligned}
$$

for some constant $C_{-r}^{\prime}$.

We first recall some probabilistic facts on $H_{-r}$-valued processes. Let $\Omega_{-r}=\mathscr{D}\left([0, \infty), H_{-r}\right)$. For $r>d / 2$, the $H_{-r}$-valued Brownian motion $W_{t}$ with covariance

$$
E\left(\left\langle g_{1}, W_{t_{1}}\right\rangle \cdot\left\langle g_{2}, W_{t_{2}}\right\rangle\right)=\left(t_{1} \wedge t_{2}\right) \cdot\left\langle g_{1}, g_{2}\right\rangle
$$


$g_{1}, g_{2} \in H_{r}$, is well-defined on $\Omega_{-r}$ (cf. [12] ch. 3, th. 3.1). We shall use the following tightness criterion on $\Omega_{-r}, r>d / 2$ : a sequence of processes $\zeta_{t}^{n}$ with laws $P^{n}$ on $\mathscr{D}\left([0, T], H_{-r}\right)$ is tight if

(i) there exists $r^{\prime}, d / 2<r^{\prime}<r$, such that

for each $\varepsilon>0$ we find $K>0$ with

and

$$
\sup _{n} P^{n}\left\{\sup _{s \leqq T}\left\|\zeta_{s}^{n}\right\|_{-r^{\prime}}^{2} \geqq K\right\} \leqq \varepsilon,
$$

(ii) for all $g \in H_{r}, \varepsilon>0, \eta>0$ there exists $\delta>0$ such that

$$
\sup _{n} \sup _{0 \leqq \tau_{1} \leqq \tau_{2} \leqq\left(\tau_{1}+\delta\right) \wedge T} P^{n}\left\{\left|\left\langle g, \xi_{\tau_{2}}^{n}\right\rangle-\left\langle g, \xi_{\tau_{1}}^{n}\right\rangle\right|>\eta\right\} \leqq \varepsilon .
$$

These conditions are an immediate consequence of Mitoma's result (see [20] Theorem 4.1 and Remark 1. Notice that (5.11) implies the uniform $r$-continuity of $P^{n}$ and that $\left\{\xi \in H_{-r^{\prime}} ;\|\xi\|_{-r^{\prime}}^{2}<K\right\}$ has compact closure in $H_{-r}{ }^{3}$ ).

We need to strengthen the assumptions made in $(2.9-10)$ :

(i) There exists $r_{0}>d / 2$ and a map $d G$ from $L_{b}^{\infty} \cap H_{r_{0}}$ into the space of continuous linear operators on $H_{-r_{0}}$ such that

$$
|\langle g, G(\mu)\rangle-\langle g, G(u \lambda)\rangle-\langle g, d G(u)(\mu-u \lambda)\rangle|=\|g\|_{r_{0}} o\left(\|\mu-u \cdot \lambda\|_{-_{0}}\right)
$$

uniformly in $g \in H_{r_{0}}, u \in L_{b}^{\infty} \cap H_{r_{0}}, \mu \in H_{-r_{0}}$, and

$$
\sup _{u \in L_{b}^{\infty} \cap H_{r_{0}}} \sup _{\mu \in H_{-r_{0}}}\|d G(u) \mu\|_{-r_{0}} /\|\mu\|_{-r_{0}}<+\infty .
$$

Here, $G(\mu)$ is the formal (and clear) extension of $G$ given in (3.5) as a function from $H_{-r_{0}}$ to $H_{-r_{0}}$.

(ii) $G_{0}$ and $G_{1}$ are bounded continuous functions from

$$
\mathscr{M}_{b} \cap\left\{\mu \in H_{-r_{0}} ;\|\mu\|_{-r_{0}} \leqq b C_{-r_{0}}\right\} \text { into } H_{r_{0}},
$$

and $G_{2}^{n}$ satisfies

$$
\sup _{m \in B, \mu \in \mathscr{M}_{b}^{n}}\left\|G_{2}^{n}(m, ., \mu)\right\|_{r_{0}}=o\left(N^{-1 / 2}\right) .
$$

Finally, we recall some facts on Sobolev spaces. Notice that by the interpolation theorem and Sobolev's Theorem for $r_{0}>d / 2$,

$$
\|g \cdot h\|_{r_{0}} \leqq C_{r_{0}}^{\prime}\left(\|g\|_{\infty}\|h\|_{r_{0}}+\|g\|_{r_{0}}\|h\|_{\infty}\right) \leqq C_{r_{0}}^{\prime \prime}\|g\|_{r_{0}}\|h\|_{r_{0}},
$$

so that $H_{r_{0}}$ is an algebra (cf. [24] II, 2.1). In (5.15), we used that

$$
\|g\|_{\infty} \leqq \sum_{p}|\hat{g}(p)| \leqq C_{r_{0}}^{1 / 2}\|g\|_{r_{0}}
$$

which also shows that $H_{r_{0}} \subseteq \mathscr{C}(\mathbb{T})$, if $r_{0}>d / 2$.

Moreover, for any $\mathscr{C}^{\infty}$-function $\varphi$ on $\mathbb{R}, \varphi(g) \in H_{r_{0}}$ for $g \in H_{r_{0}}$ and

$$
\|\varphi(g)\|_{r_{0}} \leqq \psi\left(\|g\|_{r_{0}}\right)
$$

\footnotetext{
${ }^{3}$ Indeed, any bounded set in $H_{-r}$, is conditionally compact in $H_{-r}$; see [29], Lemma 10, p. 169
} 
for some continuous function ${ }^{4} \psi$. In particular,

$$
\left\|\varphi\left(g_{1}\right)-\varphi\left(g_{2}\right)\right\|_{r_{0}} \leqq C_{M}\left\|g_{1}-g_{2}\right\|_{r_{0}}
$$

for all $g_{1}, g_{2}$ with $\left\|g_{1}\right\|_{r_{0}},\left\|g_{2}\right\|_{r_{0}} \leqq M$. As a consequence of (5.14-18), we obtain that the solutions $u_{t}$ and $v_{t}$ of (3.3) and (3.5) satisfy

$$
u_{t} \in L_{b}^{\infty} \cap H_{r_{0}}, \quad v_{t} \in L_{\left[0, b^{2}\right]}^{\infty} \cap H_{r_{0}},
$$

for all $t \in \mathbb{R}^{+}$as soon as $u_{0}$ and $v_{0}$ do, and are uniformly bounded in $H_{r_{0}}$.

Now, we are ready to study the asymptotics of the non-critical fluctuation processes

$$
\zeta_{t}^{n}=N^{1 / 2}\left(\sigma_{t}^{n}-u_{t}\right) \in H_{-r_{0}} .
$$

Theorem 4. We assume (5.12-14), $\sigma_{0}^{n} \in \mathscr{M}_{b}^{n}, u_{0} \in L_{b}^{\infty} \cap H_{r_{0}}, v_{0} \in L_{\left[0, b^{2}\right]}^{\infty} \cap H_{r_{0}}$ and that $\zeta_{0}^{n}=N^{1 / 2}\left(\sigma_{0}^{n}-u_{0}\right)$ converge in law to $\zeta_{0} \in H_{-r_{0}}$ with

$$
\sup _{n} E^{n}\left\|\zeta_{0}^{n}\right\|_{-r_{0}}^{2}<\infty \text {. }
$$

Let $r>r_{0}$. Then on $\mathscr{D}\left([0, T], H_{-r}\right)$, the fluctuation processes $\zeta_{t}^{n}(5.20)$ converge in law to the process $\zeta_{t}$ satisfying

$$
d \zeta_{t}=d G\left(u_{t}\right) \zeta_{t} d t+\left(B\left(u_{t}, v_{t}\right)\right)^{1 / 2} \cdot d W_{t}
$$

with the $H_{-r}$-valued Brownian motion $W_{t}$ from (5.9) and

$$
\begin{aligned}
B(u, v)(x)= & \exp \left\{G_{0}(x, u)+\gamma\left(G_{1}(x, u)\right)\right\} \\
& \cdot\left[\gamma^{\prime \prime}\left(G_{1}(x, u)\right)+\left(\gamma^{\prime}\right)^{2}\left(G_{1}(x, u)\right)-2 u(x) \cdot \gamma^{\prime}\left(G_{1}(x, u)\right)+v(x)\right] .
\end{aligned}
$$

Proof. We first notice that (5.22) implies

$$
d\left\langle g, \zeta_{t}\right\rangle=\left\langle\left(d G\left(u_{t}\right)\right)^{*} g, \zeta_{t}\right\rangle d t+\left\langle g \cdot B\left(u_{t}, v_{t}\right)^{1 / 2}, d W_{t}\right\rangle .
$$

Therefore, if $T_{s, t}^{*}$ is the semigroup on $H_{r_{0}}$ with generator $d G\left(u_{t}\right)^{*}$, the adjoint of $d G\left(u_{t}\right)$, we have for $0 \leqq s \leqq t \leqq T$ :

$$
P\left(\left\langle g, \zeta_{t}\right\rangle \in d y \mid \zeta_{s}\right)=p\left(\int_{s}^{t}\left\langle T_{\tau, t}^{*} g, B\left(u_{\tau}, v_{\tau}\right) \cdot T_{\tau, t}^{*} g\right\rangle d \tau, y-\left\langle T_{s, t}^{*} g, \zeta_{s}\right\rangle\right) d y
$$

where $p(t, y)=(2 \pi t)^{-1 / 2} \exp \left(-y^{2} / 2 t\right)$ is the heat kernel.

This shows that the process $\zeta_{s}$ is uniquely determined by the following martingale problem: for $f \in \mathscr{C}_{b}^{2}\left(\mathbb{R}^{k}\right)$ and $g_{i} \in H_{r_{0}}$ for $i=1, \ldots, k, k \in \mathbb{N}$, we have with $\tilde{f}(\zeta)=$ $f\left(\left\langle g_{1}, \zeta\right), \ldots,\left\langle g_{k}, \zeta\right\rangle\right)$ that

where

$$
\tilde{f}\left(\zeta_{t}\right)-\int_{0}^{t} L\left(u_{s}, v_{s}\right) \tilde{f}\left(\zeta_{s}\right) d s \text { is a } P \text {-martingale, }
$$

$$
L(u, v) \tilde{f}(\zeta)=\sum_{i=1}^{k} \widetilde{\partial_{i} f}(\zeta)\left\langle g_{i}, d G(u) \zeta\right\rangle+1 / 2 \sum_{i, j}^{k} \widetilde{\partial_{i} \partial_{j} f}(\zeta)\left\langle g_{i}, B(u, v) g_{j}\right\rangle
$$

(cf. [15] Theorem 1.4).

${ }^{4}$ Estimates $(5.17,18)$ may be obtained using Littlewood-Paley decomposition of $g$ (refer to [28]) 
a) We first prove tightness. From (2.12-13) and (3.4), we obtain the martingale decomposition

$$
\begin{aligned}
\left\langle g, \zeta_{t}\right\rangle= & \left\langle g, \zeta_{0}\right\rangle+\int_{0}^{t} N^{1 / 2} \int_{\mathbb{J}} g(x) \\
& \cdot\left[\int_{B} A^{n}\left(m, x, \sigma_{s}^{n}\right)\left(m-\sigma_{s}^{n}(x)\right) \rho(d m) \lambda^{n}(d x)-G\left(u_{s}\right)(d x)\right] d s \\
& +N^{-1 / 2} \int_{0}^{t} \int_{B \times \mathbb{J}} g(\lambda)\left(m-\sigma_{s-}^{n}(x)\right) \tilde{\Lambda}^{n}(d m, d x, d s) .
\end{aligned}
$$

Set

$$
\tau_{M}^{n}=\inf \left\{t ;\left\|\zeta_{t}^{n}\right\|_{-r_{0}}^{2} \geqq M^{2}\right\} .
$$

Then, for $t_{1}<t_{2} \leqq \tau_{M}^{n}$, Ito's formula yields

$$
\begin{aligned}
\left\|\zeta_{t_{2}}^{n}\right\|_{-r_{0}}^{2}= & \left\|\zeta_{t_{1}}^{n}\right\|_{-r_{0}}^{2} \\
& +2 N^{1 / 2} \int_{t_{1}}^{t_{2}}\left\langle\zeta_{s}^{n}, \int_{B}\left[A^{n}\left(m, ., \sigma_{s}^{n}\right)\left(m \lambda^{n}(\cdot)-\sigma_{s}^{n}(\cdot)\right) \rho(d m)-G\left(u_{s}\right)\right\rangle_{-r_{0}} d s\right. \\
& +\int_{t_{1}}^{t_{2}} \int_{B \times \mathbb{T}}\left\|\left(m-\sigma_{s}^{n}(x)\right) \delta_{x}\right\|_{-r_{0}}^{2} A^{n}\left(m, x, \sigma_{s}^{n}\right) \rho(d m) \lambda^{n}(d x) d s \\
& +\int_{t_{1}}^{t_{2}} \int_{B \times \mathbb{T}}\left(\left\|\zeta_{s-}^{n}+N^{-1 / 2}\left(m-\sigma_{s-}^{n}(x)\right) \delta_{x}\right\|_{-r_{0}}^{2}-\left\|\zeta_{s-}^{n}\right\|_{-r_{0}}^{2}\right) \tilde{\Lambda}^{n}(d m, d x, d s) .
\end{aligned}
$$

The second term of the right-hand side of (5.30) gives

$$
\begin{aligned}
\mid \text { second term } \mid= & \mid 2 N^{1 / 2} \int_{t_{1}}^{t_{2}}\left\langle\zeta_{s}^{n}, G\left(\sigma_{s}^{n}\right)-G\left(u_{s}\right)\right\rangle_{-r_{0}} d s \\
& +2 N^{1 / 2} \int_{i_{1}}^{t_{2}}\left\langle\zeta_{s}^{n}, \int\left[A _ { B } \left[A^{n}\left(m, ., \sigma_{s}^{n}\right)\left(m d \lambda^{n}-d \sigma_{s}^{n}\right)\right.\right.\right. \\
& \left.\left.\quad-A\left(m, ., \sigma_{s}^{n}\right)\left(m d \lambda-d \sigma_{s}^{n}\right)\right] d \rho\right\rangle_{-r_{0}} d s \mid \\
\leqq & 2 \int_{t_{1}}^{t_{2}}\left|\left\langle\zeta_{s}^{n}, d G\left(u_{s}\right) \zeta_{s}^{n}\right\rangle_{-r_{0}}\right|+o\left(\left\|\zeta_{s}^{n}\right\|_{-r_{0}}^{2}+1\right) d s \\
& +N^{1 / 2}\left\|\lambda^{n}-\lambda\right\|_{-r_{0}} 2 \int_{i_{1}}^{t_{2}}\left\|\zeta_{s}^{n}\right\|_{-r_{0}}\left\|\int_{B} A^{n}\left(m, ., \sigma_{s}^{n}\right) m \rho(d m)\right\|_{r_{0}} d s \\
\leqq & C_{1} / 2 \int_{t_{1}}^{t_{2}}\left(\left\|\zeta_{s}^{n}\right\|_{-r_{0}}^{2}+1\right) d s .
\end{aligned}
$$

Also the integrand of the third term of (5.30) is bounded, by $C_{1} / 2$ say. Therefore

$$
\left\|\zeta_{t \wedge \tau_{M}^{n}}^{n}\right\|_{-r_{0}}^{2} \pm C_{1} \int_{0}^{t \wedge \tau_{M}^{n}}\left(\left\|\zeta_{s}^{n}\right\|_{-r_{0}}^{2}+1\right) d s
$$

is a submartingale, respectively a supermartingale. Taking expectations of this 
supermartingale and using Gromwall's lemma together with (5.21), we get

$$
E^{n}\left(\left\|\zeta_{t}^{n}\right\|_{-r_{0}}^{2}\right)=\lim _{M \rightarrow \infty} E^{n}\left(\left\|\zeta_{t \wedge \tau_{M}^{n}}^{n}\right\|_{-r_{0}}^{2}\right) \leqq C_{2} e^{C_{1} t}
$$

for all $t \leqq T$ and $n \geqq n_{0}$, where $n_{0}$ depends only on the values in (5.7) and $(5.11-18)$. By Doob's submartingale inequality, we get

$$
\begin{aligned}
P^{n}\left\{\sup _{t \leqq T}\left\|\zeta_{t}^{n}\right\|_{-r_{0}}^{2} \geqq K\right\} & \leqq P^{n}\left\{\sup _{t \leqq T}\left(\left\|\zeta_{t}^{n}\right\|_{-r_{0}}^{2}+C_{1} \int_{0}^{t}\left(\left\|\zeta_{s}^{n}\right\|_{-r_{0}}^{2}+1\right) d s\right) \geqq K\right\} \\
& \leqq K^{-1}\left[E^{n}\left(\left\|\zeta_{T}^{n}\right\|_{-r_{0}}^{2}\right)+C_{1} \int_{0}^{T}\left(E\left(\left\|\zeta_{s}^{n}\right\|_{-r_{0}}^{2}\right)+1\right) d s\right] \\
& \leqq K^{-1}\left[2 C_{2} e^{C_{1} T}+C_{1} T\right]<\varepsilon
\end{aligned}
$$

for $K$ large enough. This shows (5.10).

Next, let $\tau_{1} \leqq \tau_{2} \leqq\left(\tau_{1}+\delta\right) \wedge T$ be stopping times and $g \in H_{r} \subset H_{r_{0}}$.

Applying similar inequalities as in (5.28), to (5.31), we find

$$
\begin{aligned}
\left\langle g, \zeta_{\tau_{2}}^{n}-\zeta_{\tau_{1}}^{n}\right\rangle= & \int_{\tau_{2}}^{\tau_{2}}\left[\left\langle g, d G\left(u_{s}\right) \zeta_{s}^{n}\right\rangle+\|g\|_{r_{0}} o\left(\left\|\zeta_{s}^{n}\right\|_{-r_{0}}+1\right)+o(1)\right] d s \\
& N^{-1 / 2} \int_{\tau_{1}}^{\tau_{2}} g(x)\left(m-\sigma_{s^{-}}^{n}(x)\right) \tilde{\Lambda}^{n}(d m, d x, d s) . \\
E^{n}\left(\left\langle g, \zeta_{\tau_{2}}^{n}-\zeta_{\tau_{1}}^{n}\right\rangle^{2}\right) \leqq & 6 E^{n}\left[( \tau _ { 2 } - \tau _ { 1 } ) \int _ { \tau _ { 1 } } ^ { \tau _ { 2 } } \left(C_{3}\|g\|_{r_{0}}^{2}\left\|\zeta_{s}^{n}\right\|_{-r_{0}}^{2}\right.\right. \\
& \left.\left.+\|g\|_{r_{0}}^{2} o\left(\left\|\zeta_{s}^{n}\right\|_{-r_{0}}^{2}+1\right)+o(1)\right) d s\right] \\
& +2 E^{n}\left[\int_{\tau_{1}}^{\tau_{2}} g^{2}(x)\left(m-\sigma_{s}^{n}(x)\right)^{2} A^{n}\left(m, x, \sigma_{s}^{n}\right) \rho(d m) \lambda^{n}(d x) d s\right] \\
\leqq & C_{4}\|g\|_{r_{0}}^{2}\left(e^{C_{1} T}+1\right) \delta^{2}+C_{5}\|g\|_{L^{\infty}}^{2} b^{2} \delta,
\end{aligned}
$$

which implies for $\delta$ sufficiently small

$$
\left.P^{n}\left(\left|\left\langle g, \zeta_{\tau_{2}}^{n}-\zeta_{\tau_{1}}^{n}\right\rangle\right|\right\rangle \eta\right) \leqq \eta^{-2} E^{n}\left(\left\langle g, \zeta_{\tau_{2}}^{n}-\zeta_{\tau_{1}}^{n}\right\rangle^{2}\right) \leqq \varepsilon
$$

This shows the tightness of the fluctuation processes $\zeta_{t}^{n}$.

b) In order to characterize the limit process of $\zeta_{t}^{n}$ by the martingale problem (5.25), we apply Ito's formula to

$$
\tilde{f}\left(\zeta_{t}^{n}\right)=f\left(\left\langle g_{1}, \zeta_{t}^{n}\right\rangle, \ldots,\left\langle g_{k}, \zeta_{t}^{n}\right\rangle\right), \quad f \in \mathscr{C}_{b}^{2}\left(\mathbb{R}^{k}\right), \quad g_{i} \in H_{r} \subset H_{r_{0}} \quad \text { for } \quad i=1, \ldots, k .
$$

We write $M_{t}^{n}$ for $M_{t}^{n}\left(f\left(\ldots, N^{1 / 2}\left\langle g_{i}, \sigma-u\right\rangle, \ldots\right)\right)$ from (2.13) and use estimates similar to (5.31).

$$
\begin{aligned}
\tilde{f}\left(\zeta_{t}^{n}\right)=\tilde{f}\left(\zeta_{0}^{n}\right)+\int_{0}^{t} \sum_{i=1}^{k}\left\{\widetilde{\partial_{i} f}\left(\zeta_{s}^{n}\right) \int_{J} g_{i}(x) N^{1 / 2}\right. \\
\\
\cdot\left[\int_{B} A^{n}\left(m, x, \sigma_{s}^{n}\right)\left(m-\sigma_{s}^{n}(x)\right) \rho(d m) \lambda^{n}(d x)-G\left(u_{s}\right) \lambda(d x)\right]
\end{aligned}
$$




$$
\begin{aligned}
& +\int_{B \times \mathbb{T}}\left[f\left(.,\left\langle g_{i}, \zeta_{s}^{n}\right\rangle+N^{-1 / 2} g_{i}(x)\left(m-\sigma_{s}^{n}(x)\right), .\right)\right. \\
& \left.-\tilde{f}\left(\zeta_{s}^{n}\right)-\widetilde{\partial_{i} f}\left(\zeta_{s}^{n}\right) N^{-1 / 2} g_{i}(x)\left(m-\sigma_{s}^{n}(x)\right)\right] \\
& \left.\cdot N A^{n}\left(m, x, \sigma_{s}^{n}\right) \rho(d m) \lambda^{n}(d x)\right\} d s+M_{t}^{n} \\
& =\tilde{f}\left(\zeta_{0}^{n}\right)+\int_{0}^{t} \sum_{i=1}^{K} \widetilde{\partial_{l} f}\left(\zeta_{s}^{n}\right)\left\langle g_{i}, d G\left(u_{s}\right) \zeta_{s}^{n}\right\rangle d s \\
& +1 / 2 \int_{0}^{t} \sum_{i j} \widetilde{\partial_{i} \partial_{j} f}\left(\zeta_{s}^{n}\right) \int_{B \times \mathbb{0}} g_{i}(x) g_{j}(x)\left(m-\sigma_{s}^{n}(x)\right)^{2} \\
& \cdot A^{n}\left(m, x, \sigma_{s}^{n}\right) \rho(d m) \lambda^{n}(d x) d s+M_{t}^{n} \\
& +\int_{0}^{t}\left[o\left(\left\|\zeta_{s}^{n}\right\|-r_{0}+1\right)+o\left(N^{1 / 2}\left\|\lambda^{n}-\lambda\right\|_{-r_{0}}\right)\right] d s
\end{aligned}
$$

from Taylor formula. Developing the term containing $\left[m-\sigma_{s}^{n}(x)\right]^{2}$, and expressing $\int_{B} m^{i} e^{n \sigma} \rho(d m), i=1,2$, in terms of $\gamma$, we obtain

$$
\begin{aligned}
\tilde{f}\left(\zeta_{t}^{n}\right)= & \tilde{f}\left(\zeta_{0}^{n}\right)+\int_{0}^{t} L\left(u_{s}, v_{s}\right) \tilde{f}\left(\zeta_{s}^{n}\right) d s+M_{t}^{n}+\int_{0}^{t}\left[o\left(N^{-1 / 2}+\left\|\zeta_{s}^{n}\right\|_{-r_{0}}+1\right)\right. \\
& +\mathcal{O}\left(N^{-r / d+1 / 2}\right)+\mathcal{O}\left(\sum_{i=0}^{1}\left\|G_{i}\left(\sigma_{s}^{n}\right)-G_{i}\left(u_{s}\right)\right\|_{r_{0}}\right. \\
& +\sum_{i, j=1}^{K} \mid\left\langle g_{i} g_{j} \exp \left\{G_{0}\left(u_{s}\right)+\gamma\left(G_{1}\left(u_{s}\right)\right)\right\}, v_{s} \lambda-\left(\sigma_{s}^{n}\right)^{2}\right\rangle \\
& \left.\left.+\left\|\sigma_{s}^{n}-u_{s} \lambda\right\|_{-r_{0}}\right)\right] d s .
\end{aligned}
$$

The last integral vanishes in the limit $n \rightarrow \infty$, and so any limit process $\zeta_{t}$ of $\zeta_{t}^{n}$ satisfies the martingale problem (5.26), which has the unique solution (5.22). This completes the proof of Theorem 4 .

We review the example of Sect. 2 in the light of the last theorem. Let

$$
G_{0}(x, \sigma)=f_{0}\left(g_{1} * \sigma(x), \ldots, g_{k} * \sigma(x)\right) .
$$

with $f_{0} \in \mathscr{C}^{\left[r_{0}\right]+1}\left(\mathbb{R}^{k}\right), g_{i} \in H_{r_{0}}$. We also assume that in $(2.16) \mathscr{J}_{j} \in H_{r_{0}}\left(\mathbb{T}^{j}\right), j=1, \ldots, q$. Then both $G_{0}$ and $G_{1}$ are continuous bounded functions from $\mathscr{M}_{b} \cap\left\{\mu \in H_{-r_{0}}\right.$, $\left.\| \mu_{-r_{0}} \leqq b C_{-r_{0}}\right\}$ into $H_{r_{0}}$. Then $G_{2}^{n}$, given in (2.19), satisfies

$$
\sup _{m \in B, \mu \in \mathscr{M}_{b}^{n}}\left\|G_{2}^{n}(m, ., \mu)\right\|_{r_{0}}=\mathcal{O}\left(N^{-1}\right) .
$$

Thus Theorem 4 applies to our example.

\section{Critical Fluctuations at the Ferromagnetic Phase Transition}

Here, we consider the special case of a translation invariant, two-body interaction without external field. In the context of our example of Sect. 2, this means 


$$
\begin{gathered}
q=2, \quad \mathscr{J}_{1}=0, \quad \mathscr{J}_{2}(x, y)=\mathscr{J}^{\prime}(x-y), \\
\nabla H(\sigma)(x)=-\mathscr{J} * \sigma(x)=\int_{\mathbb{I}} \mathscr{J}(x-y) \sigma(d y)
\end{gathered}
$$

with the symmetrization $\mathscr{J}(x)=\left(\mathscr{J}^{\prime}(x)+\mathscr{J}^{\prime}(-x)\right) / 2$. We know that if $\rho$ is symmetric and satisfies the GHS-inequality (see below), if (5.1) holds, and

$$
\hat{\mathscr{J}}(0)-\hat{\mathscr{J}}(p) \geqq \delta_{0}>0 \text { for all } p \in \mathbb{Z}^{d} \backslash\{0\},
$$

then the Gibbs states to the Hamiltonian (2.15) have a second order phase transition at the critical inverse temperature

$$
\beta_{0}=\left(\gamma^{\prime \prime}(0) \hat{\mathscr{J}}(0)\right)^{-1} \text {. }
$$

This is the first phase transition as the temperature decreases from the hightemperature region. The new phase, which appears immediately below the critical temperature $\beta_{0}^{-1}$, is ferromagnetic, i.e. it has constant non-zero magnetization. In order to study critical fluctuations of the dynamical model, we make the following assumptions:

(A1) Let $\rho$ be a symmetric measure on $\mathbb{R}$ with support contained in $[-b, b], b>0$, and let $\rho$ satisfy the GHS-condition:

$$
\gamma^{(3)}(x) \leqq 0 \quad \text { for } \quad x \in[0, \infty) .
$$

Since $\gamma$ is convex and symmetric with $\gamma^{\prime \prime}(0)>0,(6.4)$ implies that there exists $K_{0} \geqq 2$, such that

$$
\gamma(0)=\gamma^{\prime}(0)=0, \quad \gamma^{\prime \prime}(0)>0, \quad \gamma^{(3)}(0)=\cdots=\gamma^{\left(2 K_{0}-1\right)}(0)=0, \quad \gamma^{\left(2 K_{0}\right)}(0)<0 .
$$

(A2) Assume

$$
G_{0} \equiv 0, \quad G_{1}(x, \sigma)=-\beta_{0} \nabla H(\sigma)(x)=\beta_{0} \mathscr{J} * \sigma(x)
$$

with $\beta_{0}$ from (6.3) and $\mathscr{J}$ satisfying (6.2).

Moreover, we require

$$
\mathscr{J} \in H_{2 r_{0}} \text { for some } r_{0}>d\left(1-1 / K_{0}\right) \geqq d / 2,
$$

which yields by $(5.16)$

$$
\|\mathscr{J} * \sigma\|_{\infty} \leqq C_{r_{0}}\|\mathscr{J} * \sigma\|_{r_{0}} \leqq C_{r_{0}}\|\mathscr{J}\|_{2 r_{0}}\|\sigma\|_{-r_{0}}
$$

such that $G_{1}$ is a continuous bounded function from

$$
\mathscr{M}_{b} \cap\left\{\mu \in H_{-r_{0}},\|\mu\|_{-r_{0}} \leqq b C_{-r_{0}}\right\} \text { into } H_{r_{0}} .
$$

We also require

$$
\sup _{m \in B, \mu \in \mathscr{M}_{b}^{n}}\left\|G_{2}^{n}(m, ., \mu)\right\|_{r_{0}}=o\left(N^{-\left(1-1 / 2 K_{0}\right)}\right) .
$$

which is satisfied if we define $G_{2}^{n}$ by (2.19) (see 5.39)).

The critical fluctuation process is defined by

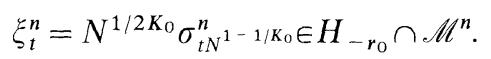


We split $\xi_{t}^{n}$ into its ferromagnetic and non-ferromagnetic components:

$$
\theta_{t}^{n}=\hat{\xi}_{t}^{n}(0) \lambda^{n}, \quad \eta_{t}^{n}=\xi_{t}^{n}-\hat{\xi}_{t}^{n}(0) \lambda^{n},
$$

$\theta_{t}^{n}$ and $\eta_{t}^{n}$ are orthogonal in $\left(H_{-r_{0}},\langle\cdot\rangle_{-r_{0}}\right)$. Notice that $\sigma^{n} \in \mathscr{M}^{n}$ implies

and

$$
\hat{\sigma}^{n}(p)=\hat{\sigma}^{n}(p+n q) \text { for all } p, q \in \mathbb{Z}^{d}
$$

$$
\widehat{\mathscr{J} * \sigma^{n}} \lambda^{n}(p)=\widehat{\mathscr{J} \lambda^{n}}(p) \hat{\sigma}^{n}(p), \quad p \in \mathbb{Z}^{d} \text {. }
$$

Theorem 5. Besides assumptions $(A 1)$ and $(A 2)$, we suppose for the starting configurations $\xi_{0}^{n}$ that

$$
\begin{gathered}
\sigma_{0}^{n} \in \mathscr{M}_{b}^{n}, \theta_{0}^{n} \text { converges in law to some } \theta_{0} \lambda ; \\
E^{n}\left\|\eta_{0}^{n}\right\|_{-r_{0}}^{2 \mathscr{K}} \leqq C_{2} \alpha_{n}^{-\mathscr{K}} \text { for all large } n
\end{gathered}
$$

where $\mathscr{K}>\mathscr{K}^{\prime}>K_{0}-1$ and $\bar{\alpha}_{n}$ an increasing (to infinity) sequence with

$$
N^{\left(1-2 / K_{0}\right)+\left(1-1 / K_{0}\right) / \mathscr{K}^{\prime}} \alpha_{n}^{-1} \rightarrow 0, \quad \text { and } \quad \alpha_{n} N^{-\left(1-1 / K_{0}\right)} \rightarrow 0 ;
$$

$$
E^{n}\left(\left\|\left(\sigma_{0}^{n}(\cdot)\right)^{2}-\gamma^{\prime \prime}(0) \lambda^{n}\right\|_{-r_{0}}^{2 \overline{\mathcal{X}}}\right) \leqq C_{3} \bar{\alpha}_{n}^{\overline{\mathcal{K}}},
$$

where $\overline{\mathscr{K}}>1$ and $\bar{\alpha}_{n}$ a sequence with

$$
N^{\left(1-1 / K_{0}\right) / \overline{\mathscr{K}}} \alpha_{n}^{-1} \rightarrow 0, \quad \alpha_{n} N^{1-1 / K_{0}} \rightarrow 0 .
$$

Let $r>r_{0}$.

Then the critical fluctuation process $\xi_{t}^{n}$ converges in law on $\mathscr{D}\left([0, T], H_{-r}\right)$ to the ferromagnetic process $\xi_{t}=\hat{\theta}_{t}(0) \lambda$, where $\hat{\theta}_{t}(0) \in \mathbb{R}$ is given by

$$
d \hat{\theta}_{t}(0)=\gamma^{\left(2 K_{0}\right)}(0) /\left[\left(2 K_{0}-1\right) ! \gamma^{\prime \prime}(0)^{2 K_{0}-1}\right] \hat{\theta}_{t}^{2 K_{0}-1}(0) d t+\left(2 \gamma^{\prime \prime}(0)\right)^{1 / 2} d w_{t},
$$

starting at $\theta_{0}$, and where $w_{t}$ is the standard Brownian motion.

Proof. (a) Tightness: we first establish the key estimate (6.25). We start with the semimartingale decomposition of $\left\langle g, \xi_{t}^{n}\right\rangle$ with $g \in H_{r_{0}}$ :

$$
\begin{aligned}
\left\langle g, \xi_{t}^{n}\right\rangle= & \left\langle g, \theta_{t}^{n}\right\rangle+\left\langle g, \eta_{t}^{n}\right\rangle=\left\langle g, \xi_{0}^{n}\right\rangle+\int_{0}^{t} N^{1-1 / 2 K_{0}} \int_{B \times \mathbb{T}} g(x) A^{n}\left(m, x, \sigma_{s N^{1-1 / K_{0}}}^{n}\right) \\
& \cdot\left(m-\sigma_{s N^{1-1 / K_{0}}}^{n}(x)\right) \rho(d m) \lambda^{n}(d x) d s+N^{-\left(1-1 / 2 K_{0}\right)} \int_{0}^{t N^{1-1 / K_{0}}} \int_{B \times \mathbb{T}} g(x) \\
& \cdot\left(m-\sigma_{s-}^{n}(x)\right) \tilde{\Lambda}^{n}(d m, d x, d s) .
\end{aligned}
$$

By Ito's formula, we get for $s<t \leqq \tau_{M}^{n}=\inf \left\{t,\left\|\xi_{t}^{n}\right\|_{-r_{0}} \geqq M\right\}$

$$
\begin{aligned}
d\left\|\eta_{s}^{n}\right\|_{-r_{0}}^{2}= & 2\left\langle\eta_{s}^{n}, N^{1-1 / 2 K_{0}} \exp \gamma\left\{\beta_{0} \mathscr{J} * \sigma_{s N^{1-1 / K_{0}}}^{n}(\cdot)\right\}\left(\gamma^{\prime}\left(\beta_{0} \mathscr{J} * \sigma_{s N^{1-1 / K_{0}}}^{n}(\cdot)\right) \lambda^{n}\right.\right. \\
& \left.\left.-\sigma_{s N^{1-1 / K_{0}}}^{n}\right)\right\rangle_{-r_{0}} d s+\int_{B \times \mathbb{T}}\left\|\left(m-\sigma_{s N^{1-1 / K_{0}}}^{n}(x)\right)\left(\delta_{x}-\lambda^{n}\right)\right\|_{-r_{0}}^{2} \\
& \cdot A^{n}\left(m, x, \sigma_{s N^{1-1 / K_{0}}}^{n}\right) \rho(d m) \lambda^{n}(d x) d s+o(1)\left[1+\left\|\eta_{s}^{n}\right\|_{-r_{0}}\right] d s+d M_{s}^{n},
\end{aligned}
$$


where

$$
\begin{aligned}
& M_{t}^{n}=\int_{0}^{t N^{1-1 / K_{0}}} \int_{B \times \mathbb{T}}\left[\left\|\eta_{s N_{-}^{n}-\left(1-1 / K_{0}\right)}^{n}+N^{-\left(1-1 / 2 K_{0}\right)}\left(m-\sigma_{s-}^{n}(x)\right)\left(\delta_{x}-\lambda^{n}\right)\right\|_{-r_{0}}^{2}\right.
\end{aligned}
$$

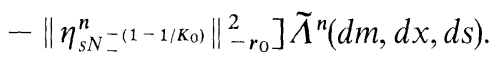

We estimate the first integral of the right-hand side of (6.21), using (6.8), (5.15-17), and

$$
\gamma^{\prime}(z)=\gamma^{\prime \prime}(0) z+\mathcal{O}\left(z^{2 K_{0}-1}\right)
$$

first term of (6.21)

$$
\begin{aligned}
= & 2\left[N^{1-1 / K_{0}}\left(\left\langle\eta_{s}^{n}, \gamma^{\prime \prime}(0) \beta_{0} \mathscr{J} * \eta_{s}^{n} \lambda^{n}-\eta_{s}^{n}\right\rangle_{-r_{0}}+\mathcal{O}\left(\left\|\eta_{s}^{n}\right\|_{-r_{0}}^{2}\left\|\xi_{s}^{n}\right\|_{-r_{0}}^{2} N^{-1 / K_{0}}\right)\right)\right. \\
& \left.+\mathcal{O}\left(\left\|\eta_{s}^{n}\right\|_{-r_{0}}\left\|\xi_{s}^{n}\right\|_{-r_{0}}^{2 K_{0}-1}\right)+O(1)\left\|\eta_{s}^{n}\right\|_{-r_{0}}\left\|\xi_{s}^{n}\right\|_{-r_{0}}\right] d s,
\end{aligned}
$$

where the scalar product $\langle., .\rangle_{-r_{0}}$ is equal to

$$
\sum_{p \notin(n \mathbb{Z})^{d}}\left(1+|p|^{2}\right)^{-r_{0}}\left|\hat{\eta}_{s}^{n}(p)\right|^{2}\left[\gamma^{\prime \prime}(0) \beta_{0} \widehat{J}^{n}(p)-1\right],
$$

since $\hat{\eta}_{s}^{n}(p)=0$ for all $p \in(n \mathbb{Z})^{d}$. From $\widehat{\mathscr{J}} \lambda^{n}(p)=\sum_{q \in \mathbb{Z}^{d}} \hat{\mathscr{J}}(p+n q)$ and $(6.2)$, we obtain the rough estimate

$$
\widehat{\mathscr{J}} \lambda^{n}(p) \leqq \hat{\mathscr{J}}(0)-7 \delta_{0} / 8, \text { for all } p \notin n \mathbb{Z}^{d} \text { and } n \geqq n_{0},
$$

which implies by $(6.3)$

$$
\gamma^{\prime \prime}(0) \beta_{0} \mathscr{J} \lambda^{n}(p)-1 \leqq-2 / 3 \gamma^{\prime \prime}(0) \beta_{0} \delta_{0}, \quad p \notin n \mathbb{Z}^{d}, \quad n \geqq n_{0} .
$$

Therefore, assuming $\mathcal{O}\left(N^{-1 / K_{0}} M^{2}\right) \leqq 1 / 8 \gamma^{\prime \prime}(0) \beta_{0} \delta_{0}$ for $n$ large, first integrand of (6.21)

$$
\leqq-N^{1-1 / K_{0}} 3 / 4 \gamma^{\prime \prime}(0) \beta_{0} \delta_{0}\left\|\eta_{s}^{n}\right\|_{-r_{0}}^{2}+C_{1}(M)\left\|\eta_{s}^{n}\right\|_{-r_{0}}
$$

for $n \geqq n_{0}(M)$. The second integrand of (6.21) is bounded by some constant $C_{2}$. With $C_{3}(M)=C_{1}(M) \cdot M+C_{2}, \delta=3 / 4 \gamma^{\prime \prime}(0) \beta_{0} \delta_{0}>0$ and $M_{s, t}^{n}=M_{t}^{n}-M_{s}^{n}$, we have for $t_{1}<t_{2} \leqq \tau_{M}^{n}, n \geqq n_{0}(M)$,

$$
\left\|\eta_{t_{2}}^{n}\right\|_{-r_{0}}^{2} \leqq\left\|\eta_{t_{1}}^{n}\right\|_{-r_{0}}^{2}-\int_{t_{1}}^{t_{2}}\left(N^{1-1 / K_{0}} \delta\left\|\eta_{s}^{n}\right\|_{-r_{0}}^{2}-C_{3}(M)\right) d s+M_{t_{1}, t_{2}}^{n}
$$

The drift term in the last member is strongly attractive to zero. To (6.25), we apply the proposition on collapsing processes, given in the appendix, with $m=N^{1-1 / K_{0}}$. Equations (6.16) and (6.15),imply (A.2) and (A.3), and since here $Y=B \times \mathbb{T}$ and

$$
\begin{aligned}
f_{t}^{n}(m, x)= & 2 N^{-\left(1-1 / 2 K_{0}\right)}\left\langle\eta_{t}^{n},\left(m-\sigma_{t N^{\left(1-1 / K_{0}\right)}}^{n}(x)\right)\left(\delta_{x}-\lambda^{n}\right)\right\rangle_{-r_{0}} \\
& +N^{-\left(2-1 / K_{0}\right)}\left\|\left(m-\sigma_{t N^{\left(1-1 / K_{0}\right)}}^{n}(x)\right)\left(\delta_{x}-\lambda^{n}\right)\right\|_{-r_{0}}^{2},
\end{aligned}
$$

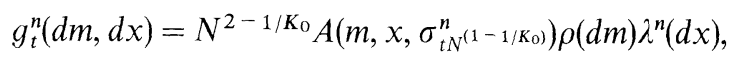

it is easy to check that

$$
\begin{aligned}
\sup \left|f_{t}^{n}(m, x)\right| & \leqq 4 b N^{-\left(1-1 / 2 K_{0}\right)} C_{-r_{0}}^{1 / 2}\left\|\eta_{t}^{n}\right\|_{-r_{0}}+b^{2} C_{-r_{0}} N^{-\left(2-1 / K_{0}\right)} \\
& \leqq C_{4}(M) N^{-\left(1-1 / 2 K_{0}\right)},
\end{aligned}
$$




$$
\int_{B \times T}\left|f_{t}^{n}(m, x)\right|^{2} g_{t}(d m, d x) \leqq C_{5}\left(\left\|\eta_{t}^{n}\right\|_{-r_{0}}^{2}+N^{-\left(2-1 / K_{0}\right)}\right),
$$

which are both sharper than required by (A.5) and (A.8). Therefore

$$
\left.P^{n}\left\{\sup _{t \leqq T \wedge \tau_{M}^{n}}\left\|\eta_{t}^{n}\right\|_{-r_{0}}^{2}>C_{6}(M) N^{\left(1-1 / K_{0}\right.}\right)^{/ / *} \alpha_{n}^{-1}\right\} \leqq \varepsilon
$$

for all large $n$. Since $C_{6}(M) \leqq N^{\left(1-1 / K_{0}\right)\left(1 / \mathscr{K}^{\prime}-1 / \mathscr{K}\right)}$ for large $n$, we find that the sets

$$
A_{n}=\left\{\sup _{t \leqq T \wedge \tau_{M}^{n}}\left\|\eta_{t}^{n}\right\|_{-r_{0}}^{2} \leqq N^{\left(1-1 / K_{0}\right) \cdot \mathscr{K}^{\prime}} \alpha_{n}^{-1} \leqq 1\right\}
$$

have $P^{n}$-probabilities greater than $1-\varepsilon$ for $n \geqq n_{0}(M, \varepsilon)$.

Similarly to $(6.21-23)$, we investigate the ferromagnetic component $\theta_{t}^{n}$ in $t_{1} \leqq$ $t_{2} \leqq T \wedge \tau_{M}^{n}$, using (5.20), (6.8) and the expression $\gamma^{\prime}(Z)=\gamma^{\prime \prime}(0) Z+\gamma^{\left(2 K_{0}\right)}(0) Z^{2 K_{0}-1} /$ $\left(2 K_{0}-1\right) !+\mathcal{O}\left(Z^{2 K_{0}}\right)$. We have

$$
\begin{aligned}
& \left\|\theta_{t_{2}}^{n}\right\|_{-r_{0}}^{2}=\left\|\theta_{t_{1}}^{n}\right\|_{-r_{0}}^{2}+2 \int_{t_{1}}^{t_{2}}\left[\mathcal{O}\left(N^{1-1 / K_{0}}\left\|\theta_{s}^{n}\right\|_{-r_{0}}\left\|\gamma^{\prime \prime}(0) \beta_{0} \mathscr{J} * \theta_{s}^{n} \lambda^{n}-\theta_{s}^{n}\right\|_{-r_{0}}\right)\right. \\
& +\left\langle\theta_{s}^{n}, \gamma^{\left(2 K_{0}\right)}(0) /\left(2 K_{0}-1\right) !\left(\beta_{0} \mathscr{J} * \xi_{s}^{n}\right)^{2 K_{0}-1} \hat{\lambda}^{n}\right\rangle_{-r_{0}} \\
& +o(1)\left\|\theta_{s}^{n}\right\|_{-r_{0}}\left\|\xi_{s}^{n}\right\|_{-r_{0}}+N^{1-1 / K_{0}}\left\langle\theta_{s}^{n},\left\{\exp \gamma\left(\beta_{0} \mathscr{J} * \sigma_{s N^{1-1 / K_{0}}}^{n}(\cdot)\right)-1\right\}\right. \\
& \left.\cdot\left\{\gamma^{\prime \prime}(0) \beta_{0} \mathscr{J} * \eta_{s}^{n} \lambda_{s}^{n}\right\}\right\rangle_{-r_{0}}+\mathcal{O}\left(N^{-1 / K_{0}}\left\|\theta_{s}^{n}\right\|_{-r_{0}}\left\|\xi_{s}^{n}\right\|^{2 K_{0}+1}\right) \\
& +\mathcal{O}\left(N^{-1 / 2 K_{0}}\left\|\theta_{s}^{n}\right\|_{-r_{0}}\left\|\xi_{s}^{n}\right\|_{-r_{0}}^{2 K_{0}}\right] d s+\int_{t_{1}}^{t_{2}} \int_{B \times \mathbb{T}}\left\|\left(m-\sigma_{s N^{1-1 / K_{0}}}^{n}(x)\right) \lambda^{n}\right\|_{-r_{0}} \\
& \cdot A^{n}\left(m, x, \sigma_{s N^{1-1 / K_{0}}}^{n}\right) \rho(d m) \lambda^{n}(d x) d s+\tilde{M}_{t_{1}, t_{2}}^{n},
\end{aligned}
$$

where

$$
\begin{aligned}
\tilde{M}_{t_{1}, t_{2}}^{n}= & \int_{t_{1} N^{1}}^{t_{2} N^{1-1 / K_{0}}} \int_{B \times \mathbb{T}}\left(\left\|\theta_{s N^{-\left(1-1 / K_{0}\right)}}^{n}+N^{-\left(1-1 / 2 K_{0}\right)}\left(m-\sigma_{s-}^{n}(x)\right) \lambda^{n}\right\|_{-r_{0}}^{2}\right. \\
& \left.-\left\|\theta_{s N^{-\left(1-1 / K_{0}\right)}}^{n}\right\|_{-r_{0}}^{2}\right) \tilde{\Lambda}^{n}(d m, d x, d s) .
\end{aligned}
$$

By (6.3) and (5.10), we have the estimates

$$
\begin{aligned}
& N^{1-1 / K_{0}}\left\|\gamma^{\prime \prime}(0) \beta_{0} \widehat{\mathscr{J} * \theta_{s}^{n} \lambda^{n}}(0) \widehat{\lambda}^{n}-\theta_{s}^{n}(0) \lambda^{n}\right\|_{-r_{0}} \\
& =N^{1-1 / K_{0}}\left|\gamma^{\prime \prime}(0) \beta_{0}\left(\mathscr{I} \lambda^{n}(0)\right) \beta_{0}\left(\widehat{\mathscr{J}} \widehat{\lambda}^{n}(0)-\widehat{\mathscr{J}}(0)\right)\right|\left\|\theta_{s}^{n}(0) \lambda^{n}\right\|_{-r_{0}} \\
& \leqq N^{1-1 / K_{0}} \gamma^{\prime \prime}(0) \beta_{0}\|\mathscr{J}\|_{r_{0}}\left\|\lambda^{n}-\lambda\right\|_{-r_{0}}\left\|\theta_{s}^{n}\right\|_{-r_{0}}=\mathcal{O}\left(N^{-r_{0}+1-1 / K_{0}}\left\|\theta_{s}^{n}\right\|_{-r_{0}}\right) \text {, } \\
& N^{1-1 / K_{0}}\left\langle\theta_{s}^{n},\left(\exp \gamma\left(\beta_{0} \mathscr{J} * \sigma_{s N^{1-1 / K_{0}}}^{n}(\cdot)\right)-1\right)\left(\gamma^{\prime \prime}(0) \beta_{0} \mathscr{J} * \eta_{s}^{n} \lambda^{n}-\eta_{s}^{n}\right)\right\rangle_{-r_{0}} \\
& =\mathcal{O}\left(N^{1-2 / K_{0}}\left\|\theta_{s}^{n}\right\|_{-r_{0}}\left\|\xi_{s}^{n}\right\|_{-r_{0}}\left\|\eta_{s}^{n}\right\|_{-r_{0}}^{2}\right),
\end{aligned}
$$


and, using $\xi_{s}^{n}=\theta_{s}^{n}+\eta_{s}^{n}$,

$$
\begin{aligned}
& \left\langle\theta_{s}^{n}, \gamma^{\left(2 K_{0}\right)}(0) /\left(2 K_{0}-1\right) !\left(\beta_{0} \mathscr{J} * \xi_{s}^{n}\right)^{2 K_{0}-1} \lambda^{n}\right\rangle_{-r_{0}} \\
& \quad=\gamma^{\left(2 K_{0}\right)}(0) /\left(2 K_{0}-1\right) ! \beta_{0}^{2 K_{0}-1} \widehat{\mathscr{J}} \lambda^{n}(0)^{2 K_{0}-1} \widehat{\theta_{s}^{n}(0)^{2 K_{0}}\left\|\lambda^{n}\right\|_{-r_{0}}^{2}} \\
& \quad+\mathcal{O}\left(\left\|\theta_{s}^{n}\right\|_{-r_{0}}^{2 K_{0}-2}\left\|\eta_{s}^{n}\right\|_{-r_{0}}^{2}+\left\|\theta_{s}^{n}\right\|_{-r_{0}}\left\|\eta_{s}^{n}\right\|_{-r_{0}}^{2 K_{0}-1}\right)
\end{aligned}
$$

since $\mathscr{J} * \widehat{\eta_{s}^{n} \lambda^{n}}(0)=\widehat{\mathscr{J}} \lambda^{n}(0) \hat{\eta}_{s}^{n}(0)=0$ and (6.12) implies $\left\langle\theta_{s}^{n}, \mathscr{J} * \eta_{s}^{n} \lambda^{n}\right\rangle_{-r_{0}}=0$. The second integral in (6.32) is again bounded by $\left(t_{2}-t_{1}\right) C_{7}$ with $C_{7}$ independent of $M$. Hence

$$
\begin{aligned}
\left\|\theta_{t_{2}}^{n}\right\|_{-r_{0}}^{2} \leqq & \left\|\theta_{t_{1}}^{n}\right\|_{-r_{0}}^{2}+2 \int_{t_{1}}^{t_{2}} \gamma^{\left(2 K_{0}\right)}(0) /\left(2 K_{0}-1\right) ! \beta_{0}^{2 K_{0}-1} \widehat{\mathscr{J} \lambda^{n}}(0)^{2 K_{0}-1} \hat{\theta}_{s}^{n}(0)^{2 K_{0}}\left\|\lambda^{n}\right\|_{-r_{0}}^{2} d s \\
& +\int_{t_{1}}^{t_{2}}\left[\mathcal { O } _ { M } \left(N^{-r_{0}+1-1 / K_{0}}+\left(N^{1-2 / K_{0}}+1\right)\left\|\eta_{s}^{n}\right\|_{-r_{0}}^{2}+\left\|\eta_{s}^{n}\right\|_{-r_{0}}^{2 K_{0}-1}\right.\right. \\
& \left.\left.+N^{-1 / K_{0}}+N^{-1 / 2 K_{0}}\right)+o(1) M^{2}\right] d s+C_{7}\left(t_{2}-t_{1}\right)+\tilde{M}_{t_{1}, t_{2}}^{n}
\end{aligned}
$$

where the constants in the term $\mathcal{O}_{M}$ depend on $M$. The first condition of (6.16) shows that we can find $n_{1}(\varepsilon, M) \geqq n_{0}(\varepsilon, M)$ such that on the sets $A_{n}$ from (6.31), the second integrand in (6.37) is less than 1 for all $n \geqq n_{1}(\varepsilon, M)$, and the first integrand is non-positive, thanks to $\gamma^{\left(2 K_{0}\right)}(0)<0$. Equation (6.14) implies $P^{n}\left\{\left\|\theta_{0}^{n}\right\|_{-r_{0}}^{2} \geqq C_{8}\right\}<\varepsilon$ for $C_{8}$ large enough and for all $n$, by which, together with (6.37), we obtain for $n \geqq n_{1}(\varepsilon, M)$,

$$
\begin{aligned}
\left\{\left\|\theta_{0}^{n}\right\|_{-r_{0}}^{2} \leqq C_{8}\right\} & \cap A_{n} \cap\left\{\sup _{t \leqq T \wedge \tau_{M}^{n}}\left\|\theta_{t}^{n}\right\|_{-r_{0}}^{2} \geqq T\left(C_{7}+1\right)+C_{8}+C_{9}\right\} \\
& \subseteq\left\{\sup _{t \leqq T \wedge \tau_{M}^{n}} M_{t} \geqq C_{9}^{n}\right\} .
\end{aligned}
$$

But

$$
P^{n}\left\{\sup _{t \leqq T \wedge \tau_{M}^{n}} \tilde{M}_{t}^{n} \geqq C_{9}\right\} \leqq C_{9}^{-2} E\left(\tilde{M}_{T \wedge \tau_{M}^{n}}^{n}\right) \leqq C_{9}^{-2} C_{10} \leqq \varepsilon,
$$

where $C_{10}$ is independent of $n$ and $M$ and $C_{9} \geqq\left(C_{10} / \varepsilon\right)^{1 / 2}$. By (6.31) and (6.38-39), we finally get for $M>1+T\left(C_{7}+1\right)+C_{8}+C_{9}$

$$
\begin{aligned}
\left\{\tau_{M}^{n} \leqq T\right\} & =\left\{\sup _{t \leqq T \wedge \tau_{M}^{n}}\left\|\xi_{t}^{n}\right\|_{-r_{0}}^{2} \geqq M\right\} \subseteq\left\{\sup _{t \leqq T \wedge \tau_{M}^{n}}\left\|\eta_{t}^{n}\right\|_{-r_{0}}^{2}>1\right\} \cup \Omega \backslash A_{n} \cup\left\{\left\|\theta_{0}^{n}\right\|_{-r_{0}}^{2}\right. \\
& \left.\geqq C_{8}\right\} \cup\left\{\left\|\theta_{0}^{n}\right\|_{-r_{0}}^{2} \leqq C_{8}\right\} \cap A_{n} \cap\left\{\sup _{t \leqq T \wedge \tau_{M}^{n}}\left\|\theta_{t}^{n}\right\|_{-r_{0}}^{2} \geqq C_{8}+T\left(C_{7}+1\right)\right\},
\end{aligned}
$$

which show $P^{n}\left\{\tau_{M}^{n} \leqq T\right\} \leqq 4 \varepsilon$.

Hence, the condition (5.10) is satisfied. In order to establish (5.11) for $\xi_{t}^{n}$, it is enough to show it for $\theta_{t}^{n}$, since (6.30) and (6.40) show that the sequence of processes $\eta_{t}^{n}$ converges in law to $\eta_{t}=0$. 
Thus, we have, for $0 \leqq \tau_{1} \leqq \tau_{2} \leqq\left(\tau_{1}+\delta\right) \wedge T$,

$$
\begin{aligned}
\left\langle g, \theta_{\tau_{2}}^{n}-\theta_{\tau_{1}}^{n}\right\rangle= & \widehat{g} \lambda^{n}(0) \int_{\tau_{1}}^{\tau_{2}} N^{1-1 / K_{0}}\left[m-\sigma_{s N^{1-1 / K_{0}}}^{n}(x)\right] \\
& \cdot A^{n}\left(m, x, \sigma_{s N^{1-1 / K_{0}}}^{n}\right) \rho(d m) \lambda^{n}(d x) d s+\hat{M}_{\tau_{1}, \tau_{2}}^{n},
\end{aligned}
$$

with

$$
\hat{M}_{\tau_{1}, \tau_{2}}^{n}=\widehat{g \hat{\lambda}^{n}}(0) \int_{\tau_{1} N^{1}}^{\tau_{2} N^{1-1 / K_{0}}} N^{1 / K_{0}} N^{-\left(1-1 / 2 K_{0}\right)}\left[m-\sigma_{s-}^{n}(x)\right] \tilde{\Lambda}^{n}(d m, d x, d s) .
$$

After the same expansion of the first integral as in $(6.32-37)$, we have for large $n$

$$
\mathbb{1}_{\left\{\tau_{M}^{n}>T\right\}}\left\langle g, \theta_{\tau_{2}}^{n}-\theta_{\tau_{1}}^{n}\right\rangle^{2} \leqq C_{9}(M)\left(\tau_{2}-\tau_{1}\right)^{2}+2\left(\hat{M}_{\tau_{1}, \tau_{2}}^{n}\right)^{2}
$$

such that by $(6.40)$

$$
\begin{aligned}
P^{n}\left\{\left|\left\langle g, \theta_{\tau_{2}}^{n}-\theta_{\tau_{1}}^{n}\right\rangle\right|>\eta\right\} & \leqq 4 \varepsilon+P^{n}\left\{\left|\mathbb{1}_{\left\{\tau_{M}^{n}>T\right\}}\left\langle g, \theta_{\tau_{2}}^{n}-\theta_{\tau_{1}}^{n}\right\rangle\right|>\eta\right\} \\
& \leqq 4 \varepsilon+C_{9}(M) \delta^{2} / \eta^{2}+C_{10} \delta / \eta^{2} \leqq 5 \varepsilon
\end{aligned}
$$

for $n \geqq n_{2}(\varepsilon, M)$ and $\delta$ sufficiently small. This completes the proof of the tightness of the critical fluctuation process $\xi^{n}$.

b) Before we can characterize the limit process of $\xi^{n}$, we need a precise control for the convergence of the (speeded-up) process $\left(\sigma_{t}^{n}\right)^{2}$ given by (3.3):

$$
X_{t}^{n}=\left(\sigma_{t N^{1-1 / K_{0}}}^{n}\right)^{2}-\gamma^{\prime \prime}(0) \lambda^{n} \in H_{-r_{0}} .
$$

We claim that for some $n(\varepsilon)$

$$
\sup _{n \geqq n(\varepsilon)} P^{n}\left\{\sup _{t \leqq T \wedge \tau_{M}^{n}}\left\|X^{n}\right\|_{-r_{0}}^{2}>N^{\left(1-1 / K_{0}\right) / 2 \cdot \bar{k}} \alpha_{n}^{-{ }^{-1 / 2}}\right\} \leqq \varepsilon .
$$

Using Ito's formula for $\left\|X_{t}^{n}\right\|_{-r_{0}}^{2}$, and computing Sobolev-norm estimates as above, we get for $\tau_{1} \leqq \tau_{2} \leqq \tau_{M}^{n}$ :

$$
\begin{aligned}
\left\|X_{t_{2}}^{n}\right\|_{-r_{0}}^{2}= & \left\|X_{t_{1}}^{n}\right\|_{-r_{0}}^{2}-\int_{t_{1}}^{t_{2}} 2 N^{1-1 / K_{0}}\left\|X_{s}^{n}\right\|_{-r_{0}}^{2} \\
& \cdot\left[1+o\left(N^{-\left(1-1 / K_{0}\right)}\right)+\mathcal{O}\left(N^{-1 / K_{0}}\left\|\xi_{s}^{n}\right\|_{-r_{0}}^{2}\right)\right] d s \\
& +\mathcal{O}\left(N^{-1 / K_{0}}\right)\left(t_{2}-t_{1}\right)+M_{t_{1}, t_{2}}^{\prime n} \\
\leqq & \left\|X_{t_{1}}^{n}\right\|_{-r_{0}}^{2}-\int_{t_{1}}^{t_{2}}\left(N^{1-1 / K_{0}}\left\|X^{n}\right\|_{-r_{0}}^{2}+1\right) d s+M_{t_{1}, t_{2}}^{\prime n}
\end{aligned}
$$

for $t_{2} \leqq \tau_{M}^{n}$ and $n \geqq n(M)$. Applying the proposition on collapsing processes to $\left\|X_{t \wedge \tau_{M}^{n}}^{n}\right\|_{-r_{0}}^{2}$ with $\bar{\alpha}_{n}$ from (6.18), we see that (6.17) corresponds to (A.3) and (6.49) to (A.4). Here,

and

$$
\begin{aligned}
f_{t}^{n}(m, x)= & 2 N^{-1}\left\langle X_{t}^{n},\left[m^{2}-\left(\sigma_{t N^{1-1 / K_{0}}}^{n}(x)\right)^{2}\right] \delta_{x}\right\rangle_{-r_{0}} \\
& +N^{-2}\left\|\left[m^{2}-\left(\sigma_{t N^{1-1 / K_{0}}}^{n}(x)\right)^{2}\right] \delta_{x}\right\|_{-r_{0}}^{2}=\mathcal{O}\left(N^{-1}\right),
\end{aligned}
$$

$$
g^{n}(d m, d x)=N^{2-1 / K_{0}} A^{n}\left(m, x, \sigma_{t N^{1-1 / K_{0}}}^{n}\right) \rho(d m) \lambda^{n}(d x),
$$


such that (A.5) and (A.8) are also satisfied. Therefore, the proposition on collapsing processes implies (6.46).

We now compute the limit of $\xi_{t}^{n}$. It is enough to compute the limit of the one dimensional process $\hat{\xi}_{t}^{n}(0)=\hat{\theta}_{t}^{n}(0)$, since $\xi_{t}^{n}=\theta_{t}^{n}+\eta_{t}^{n}$, where $\eta_{t}^{n}$ converges to 0 , and $\theta_{t}^{n}=\hat{\theta}_{t}^{n}(0) \lambda^{n}=\hat{\theta}_{t}^{n}(0)\left[\lambda^{n}-\lambda\right]+\hat{\theta}_{t}^{n}(0) \lambda$ with $\left\|\hat{\theta}_{t}^{n}(0)\left[\lambda^{n}-\lambda\right]\right\|_{-r_{0}}=\mathcal{O}\left(N^{1 / K_{0}-r_{0}}\right)$ going to 0 . Let $f \in \mathscr{C}_{b}^{2}(\mathbb{R})$, and

$$
\begin{aligned}
\hat{\tau} & =\tau_{M}^{n} \wedge \inf \left\{t ;\left\|\eta_{t}^{n}\right\|_{-r_{0}}^{2}>N^{\left(1-1 / K_{0}\right) / \mathscr{K}^{\prime}} \alpha_{n}^{-1}\right\} \\
& \wedge \inf \left\{t ;\left\|X_{t}^{n}\right\|_{-r_{0}}^{2}>N^{\left.\left(1-1 / K_{0}\right) / 2 \overline{\mathscr{K}}_{\alpha_{n}} \bar{\alpha}_{n}^{-1 / 2}\right\} ;}\right.
\end{aligned}
$$

from $(6.30-31,40,46)$, we have $P^{n}\{\hat{\tau} \leqq T\} \leqq 6 \varepsilon$. Define the martingale

$$
\begin{aligned}
M_{t_{1}, t_{2}}^{f, n}= & \int_{t_{1} N^{1-1 / K_{0}}}^{t_{2} N^{1-1 / K_{0}}} \int_{-\pi}\left[f\left(\hat{\xi}_{s N_{-}^{-\left(1-1 / K_{0}\right.}}^{n}(0)+N^{-\left(1-1 / 2 K_{0}\right)}\left[m-\sigma_{s_{-}}^{n}(x)\right]\right)\right. \\
& \left.-\int\left(\hat{\xi}_{s N_{-}^{-}\left(1-1 / K_{0}\right)}^{n}(0)\right)\right] \tilde{\Lambda}^{n}(d m, d x, d s) .
\end{aligned}
$$

Using $(2.12,13)$, we write Ito's formula for $f\left(\hat{\theta}_{t}^{n}(0)\right)=f\left(\hat{\xi}_{t}^{n}(0)\right), t_{1} \leqq t_{2} \leqq \hat{\tau}$ :

$$
\begin{aligned}
& f\left(\hat{\xi}_{t_{2}}^{n}(0)\right)=f\left(\hat{\xi}_{t_{1}}^{n}(0)\right)+\int_{t_{1}}^{t_{2}} f^{\prime}\left(\hat{\xi}_{s}^{n}(0)\right) \int_{B \times \mathbb{I}}\left\{m-\sigma_{s N^{1-1 / K_{0}}}^{n}(x)\right\} N^{1-1 / 2 K_{0}} \\
& \cdot A^{n}\left(m, x, \sigma_{s N^{1-1 / K_{0}}}^{n}\right) \rho(d m) \hat{\lambda}^{n}(d x) d s
\end{aligned}
$$

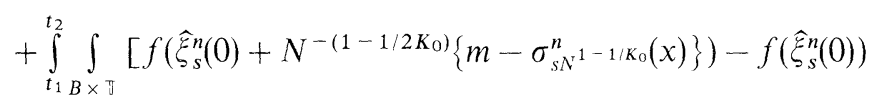

$$
\begin{aligned}
& \left.-f^{\prime}\left(\hat{\xi}_{s}^{n}(0)\right)\left\{m-\sigma_{s N^{1-1 / K_{0}}}^{n}(x)\right\} N^{-\left(1-1 / 2 K_{0}\right)}\right] \\
& \cdot N^{2-1 / K_{0}} A^{n}\left(m, x, \sigma_{s N^{1-1 / K_{0}}}^{n}\right) \rho(d m) \lambda^{n}(d x) d s+M_{t_{1}, t_{2}}^{f, n} \text {. } \\
& =f\left(\hat{\xi}_{t_{1}}^{n}(0)\right)+\int_{t_{1}}^{t_{2}}\left(f^{\prime}\left(\hat{\xi}_{s}^{n}(0)\right) N^{1-1 / 2 K_{0}}\right. \\
& \left.\cdot\left\langle\exp \gamma\left(\beta_{0} J * \sigma_{s N^{1-1 / K_{0}}}^{n}\right), \gamma^{\prime}\left(N^{-1 / 2 K_{0}} \beta_{0} J * \eta_{s}^{n}\right) \lambda^{n}-\xi_{s}^{n} N^{-1 / 2 K_{0}}\right\rangle+o(1)\right) d s \\
& +\int_{t_{1}}^{t_{2}}\left[1 / 2 f^{\prime \prime}\left(\hat{\xi}_{s}^{n}(0)\right) \int_{B \times \mathbb{T}}\{m-\sigma(x)\}^{2} A\left(m, x, \sigma_{s N^{1-1 / K_{0}}}^{n}\right) \rho(d m) \lambda^{n}(d x)\right. \\
& +o(1)] d s+M_{t_{1}, t_{2}}^{f, n},
\end{aligned}
$$

using Taylor's formula for the term between [ ] and replacing $A^{n}$ with $A$. In the first integrand of (6.52), we expand $\gamma^{\prime}$ and the exponential term as in (6.31-37), and we conclude that this integrand is equal to

$$
\begin{aligned}
& f^{\prime}\left(\hat{\xi}_{s}^{n}(0)\right) \gamma^{\left(2 K_{0}\right)}(0) /\left(2 K_{0}-1\right) !\left[\beta_{0} J \lambda^{n}(0) \hat{\theta}_{s}^{n}(0)\right]^{2 K_{0}-1} \\
& +\mathcal{O}_{M}\left(N^{1-2 / K_{0}}\left\|\eta_{s}^{n}\right\|_{-r_{0}}^{2}+\left\|\eta_{s}^{n}\right\|_{-r_{0}}\right)+o_{M}(1) .
\end{aligned}
$$

This yields for $t \leqq \hat{\tau}$,

$$
\begin{aligned}
f\left(\hat{\theta}_{t_{2}}^{n}(0)\right)= & f\left(\hat{\theta}_{t_{1}}^{n}(0)\right)+\int_{t_{1}}^{t_{2}} f^{\prime}\left(\hat{\theta}_{s}^{n}(0)\right) \gamma^{\left(2 K_{0}\right)}(0) /\left(2 K_{0}-1\right) !\left[\beta_{0} \hat{\mathscr{J}}(0) \hat{\theta}_{s}^{n}(0)\right]^{2 K_{0}-1} d s \\
& +1 / 2 \int_{t_{1}}^{t_{2}} f^{\prime \prime}\left(\hat{\theta}_{s}^{n}(0)\right) 2 \gamma^{\prime \prime}(0) d s+o_{M}(1)+M_{t_{1}, t_{2}}^{f, n} .
\end{aligned}
$$


From (6.52) and (2.14), $\sup _{n} \mathbb{E}^{n}\left(M_{t_{1}, t_{2}}^{n, f}\right)^{2} \leqq C_{11}\left(t_{2}-t_{1}\right)$, which shows that the martingales $M_{t_{1}, t_{2}}^{n, f}$ are uniformly integrable; we then conclude that any limit process $\hat{\theta}_{t}(0)$ of $\hat{\theta}_{t}^{n}(0)$ solves the martingale problem:

$$
\begin{aligned}
& f\left(\hat{\theta}_{t}(0)\right)-f\left(\hat{\theta}_{t}(0)\right)-\int_{0}^{t} f^{\prime}\left(\hat{\theta}_{s}(0)\right) \gamma^{\left(2 K_{0}\right)}(0) /\left(2 K_{0}-1\right) !\left[\beta_{0} \hat{\mathscr{J}}(0) \hat{\theta}_{s}(0)\right]^{2 K_{0}-1} d s \\
& -1 / 2 \int_{t_{1}}^{t_{2}} f^{\prime \prime}\left(\hat{\theta}_{s}(0)\right) 2 \gamma^{\prime \prime}(0) d s \quad \text { is a martingale, }
\end{aligned}
$$

which is equivalent to (6.19). This completes the proof of Theorem 5 .

The unique invariant probability measure of the process $\hat{\theta}_{t}(0) \in \mathbb{R}$ from $(6.19)$ is

$$
v_{1}(d x)=\exp \left(\gamma^{\left(2 K_{0}\right)}(0) /\left[2\left(2 K_{0}\right) !\left(\gamma^{\prime \prime}(0)\right)^{2 K_{0}}\right] x^{2 K_{0}}\right) d x / Z_{1}
$$

where $Z_{1}$ is the normalization constant.

\section{Critical Fluctuations at an Antiferromagnetic Phase Transition}

Instead of the critical fluctuations at the ferromagnetic phase transition, we now study critical fluctuations at the point of an antiferromagnetic transition with frequency $p_{0} \neq 0$. This means that instead of (6.2) and (6.3), we now have

$$
\hat{\mathscr{J}}\left(p_{0}\right)=\hat{\mathscr{J}}\left(-p_{0}\right)>0 \quad \text { and } \quad \hat{\mathscr{J}}\left(p_{0}\right)-\hat{\mathscr{J}}(q) \geqq \delta_{0}>0 \quad \text { for all } \quad q \in \mathbb{Z}^{d} \backslash\left\{ \pm p_{0}\right\},
$$

and

$$
\beta_{p_{0}}=\left(\gamma^{\prime \prime}(0) \hat{\mathscr{J}}\left(p_{0}\right)\right)^{-1} \text {. }
$$

In addition, we strengthen assumption (A1) of the last section by requiring

$$
\gamma^{(4)}(0)<0
$$

i.e. $K_{0}=2$ in (6.5). For example, this is true for Ising spins with

$$
\rho=\left(\delta_{1}+\delta_{-1}\right) / 2, \text { where } \gamma^{\prime \prime}(0)=1 \text { and } \gamma^{(4)}(0)=-2 \text {. }
$$

We keep the assumption (A2) of the last section with $K_{0}=2$. We now split the critical fluctuation process

$$
\xi_{t}^{n}=N^{1 / 4} \sigma_{t N^{1 / 2}}^{n}
$$

into the $p_{0}$-antiferromagnetic component and its complement

$$
\begin{aligned}
& \varphi_{t}^{n}(d x)=2\left[\operatorname{Re}\left(\widehat{\xi_{t}^{n}}\left(p_{0}\right)\right) \cos \left(2 \pi p_{0} x\right)+\operatorname{Im}\left(\widehat{\xi}_{T}^{n}\left(p_{0}\right)\right) \sin \left(2 \pi p_{0} x\right)\right] \lambda^{n}(d x) . \\
& \psi_{t}^{n}(d x)=\xi_{t}^{n}(d x)-\varphi_{t}^{n}(d x) .
\end{aligned}
$$

Theorem 6. Let (7.1-2), (A1) with (7.3) and (A.2) hold. For the starting configurations, we assume

(i) $\quad \sigma_{0}^{n} \in \mathscr{M}_{b}^{n}$, and $\widehat{\varphi_{0}^{n}}\left(p_{0}\right)=N^{1 / 4} \widehat{\sigma_{0}^{n}}\left(p_{0}\right)$ converges in law to some $\hat{\varphi}_{0}\left(p_{0}\right)$;

(ii) for some $\kappa>1$ and an increasing sequence $\alpha_{n}$ with

$$
N^{1 / 2 *} \alpha_{n}^{-1} \rightarrow 0 \text {, and } N^{-1 / 2} \alpha_{n} \rightarrow 0,
$$


we have

$$
E^{n}\left\|\psi_{0}^{n}\right\|_{-r_{0}}^{2 \kappa} \leqq C_{1} \alpha_{n}^{-\kappa}
$$

and

$$
E^{n}\left(\left\|\left(\left(\sigma_{0}^{n}(\cdot)\right)^{2}-\gamma^{\prime \prime}(0)\right) \lambda^{n}\right\|_{-r_{0}}^{2 \kappa}\right) \leqq C_{2} \alpha_{n}^{-\kappa}
$$

for all large $n$. Then the critical fluctuation process converges in law to the $p_{0^{-}}$ antiferromagnetic process,

$$
\varphi_{t}(d x)=2\left[\operatorname{Re}\left(\hat{\varphi}_{t}\left(p_{0}\right)\right) \cos \left(2 \pi p_{0} x\right)+\operatorname{Im}\left(\hat{\varphi}_{t}\left(p_{0}\right) \sin \left(2 \pi p_{0} x\right)\right] \lambda(d x),\right.
$$

where $\hat{\varphi}_{t}\left(p_{0}\right) \in \mathbb{C}$ satisfies the complex diffusion equation

$$
d \hat{\varphi}_{t}\left(p_{0}\right)=\gamma^{(4)}(0) / 2 \gamma^{\prime \prime}(0)^{3}\left|\hat{\varphi}_{t}\left(p_{0}\right)\right|^{2} \hat{\varphi}_{t}\left(p_{0}\right) d t+\left(2 \gamma^{\prime \prime}(0)\right)^{1 / 2} d w_{t}^{c},
$$

starting at $\hat{\varphi}_{0}\left(p_{0}\right)$. Here, $w_{t}^{c}$ denotes a complex-valued Brownian motion.

Proof. Since the proof follows the same lines as that of the last section, we will give only the main estimates. Like in (6.21-25), we obtain for $t_{1}<t_{2}<\tau_{M}^{n}=$ $\inf \left\{t ;\left\|\xi_{t}^{n}\right\|-r_{0} \geqq M\right\}$,

$$
\begin{aligned}
\left\|\psi_{t_{2}}^{n}\right\|_{-r_{0}}^{2}= & \left\|\psi_{t_{1}}^{n}\right\|_{-r_{0}}^{2}+2 \int_{i_{1}}^{t_{2}}\left[N^{1 / 2}\left\langle\psi_{s}^{n}, \gamma^{\prime \prime}(0) \beta_{p_{0}} \mathscr{J} * \psi_{s}^{n} \lambda^{n}-\psi_{s}^{n}\right\rangle_{-r_{0}}+\mathcal{O}\left(\left\|\psi_{s}^{n}\right\|_{-r_{0}} M^{3}\right)\right. \\
& \left.+o(1)\left\|\psi_{s}^{n}\right\|_{-r_{0}}\left\|\xi_{s}^{n}\right\|_{-r_{0}}\right] d s+\mathcal{O}(1)\left(t_{2}-t_{1}\right)+Q_{t_{1} t_{2}}^{n}
\end{aligned}
$$

with the martingale

$$
\begin{aligned}
Q_{t_{1}, t_{2}}^{n}= & \int_{t_{3} N^{1 / 2}}^{t_{2} N^{1 / 2}} \int_{B \times \mathbb{T}}\left[\| \psi_{s N_{-}^{-1 / 2}}^{n}+N^{-3 / 4}\left(m-\sigma_{s_{-}}^{n}(x)\right)\left(\delta_{x}-2 \cos \left(2 \pi p_{0}(x-\cdot)\right) \lambda^{n} \|_{-r_{0}}^{2}\right.\right. \\
& \left.-\left\|\psi_{s N_{-}^{1 / 2}}^{n}\right\|_{-r_{0}}^{2}\right] \tilde{\Lambda}^{n}(d m, d x, d s) .
\end{aligned}
$$

Using (7.1-2), we have

and

$$
\gamma^{\prime \prime}(0) \beta_{p_{0}} \widehat{\mathscr{J}} \lambda^{n}(q)-1 \leqq-1 / 2 \gamma^{\prime \prime}(0) \beta_{p_{0}} \delta_{0} \quad \text { for all } q \in(\mathbb{Z} / n \mathbb{Z})^{d} \backslash\left\{ \pm p_{0}\right\},
$$

$$
\left\|\psi_{t_{2}}^{n}\right\|_{-r_{0}}^{2} \leqq\left\|\psi_{t_{1}}^{n}\right\|_{-r_{0}}^{2}+\int_{t_{1}}^{t_{2}}\left(-N^{1 / 2} \gamma^{\prime \prime}(0) \beta_{p_{0}} \delta_{0}\left\|\psi_{s}^{n}\right\|_{-r_{0}}^{2}+C_{3}(M)\right) d s+Q_{t_{1}, t_{2}}^{n} .
$$

By (7.8-9) and estimates, similar to (6.28-29), we see that the assumptions of the proposition of the Appendix with $m=N^{1 / 2}$ are satisfied, so that

$$
P^{n}\left\{\sup _{t \leqq T \wedge \tau_{M}^{n}}\left\|\psi_{t}^{n}\right\|_{-r_{0}}^{2}>N^{1 / 4 \kappa} \alpha_{n}^{-1 / 2}\right\} \leqq P^{n}\left\{\sup _{t \leqq T \wedge \tau_{M}^{n}}\left\|\psi_{t}^{n}\right\|_{-r_{0}}^{2}>C_{4}(M) N^{1 / 2 \kappa} \alpha_{n}^{-1}\right\}<\varepsilon
$$

for all $n \geqq n_{0}(M, \varepsilon)$. For the $p_{0}$-antiferromagnetic component and $t_{1}<t_{2}<\tau_{M}^{n}$, we get the estimate

$$
\begin{aligned}
\left\|\varphi_{t_{2}}^{n}\right\|_{-r_{0}}^{2}= & \left\|\varphi_{t_{1}}^{n}\right\|_{-r_{0}}^{2}+2 \int_{t_{1}}^{t_{2}}\left[\mathcal{O}\left(N^{-r_{0}+1 / 2}\left\|\varphi_{\mathrm{s}}^{n}\right\|_{-r_{0}}^{2}\right)\right. \\
& +\left\langle\varphi_{s}^{n}, \gamma^{(4)}(0) / 3 !\left(\beta_{p_{0}} \mathscr{J} * \varphi_{s}^{n}\right)^{3} \lambda^{n}\right\rangle_{-r_{0}}
\end{aligned}
$$




$$
\begin{aligned}
& +\mathcal{O}\left(\left\|\varphi_{s}^{n}\right\|_{-r_{0}}\left\|\psi_{s}^{n}\right\|_{-r_{0}}\left\|\xi_{s}^{n}\right\|_{-r_{0}}^{2}\right)+\mathcal{O}\left(N^{-1 / 2}\left\|\varphi_{s}^{n}\right\|_{-r_{0}}\left\|\xi_{s}^{n}\right\|_{-r_{0}}^{5}\right) \\
& \left.+o(1)\left\|\varphi_{s}^{n}\right\|_{-r_{0}}\left\|\xi_{s}^{n}\right\|_{-r_{0}}\right] d s+\mathcal{O}(1)\left(t_{2}-t_{1}\right)+\widetilde{Q}_{t_{1}, t_{2}}^{n}, \\
\widetilde{Q}_{t_{1}, t_{2}}^{n}= & \int_{t_{1} N^{1 / 2}}^{t_{2} N_{B \times \mathbb{T}}^{1 / 2}}\left[\left\|\varphi_{s N^{-1 / 2}}^{n}+N^{-3 / 4}\left(m-\sigma_{s}^{n}(x)\right) 2 \cos \left(2 \pi p_{0}(x-\cdot)\right) \lambda^{n}\right\|_{-r_{0}}^{2}\right. \\
& \left.-\left\|\varphi_{s N^{1 / 2}}^{n}\right\|_{-r_{0}}^{2}\right] \tilde{\Lambda}^{n}(d m, d x, d s) .
\end{aligned}
$$

We calculate

$$
\begin{aligned}
& \gamma^{(4)}(0) / 3 ! \beta_{p_{0}}^{3}\left\langle\varphi_{s}^{n},\left(\mathscr{J} * \varphi_{s}^{n}\right)^{3} \lambda^{n}\right\rangle=\gamma^{(4)}(0) / 3 ! \beta_{p_{0}}^{3} \widehat{\mathscr{J} \lambda^{n}\left(p_{0}\right)^{3}\left|\widehat{\varphi}_{s}^{n}\left(p_{0}\right)\right|^{4}} \\
& \cdot \sum_{q \in(n \mathbb{Z})^{d}}\left(1+\left|p_{0}+q\right|^{2}\right)^{-r_{0}}<0,
\end{aligned}
$$

since $\gamma^{(4)}(0)<0$. Therefore, using (7.17), we find that for $n \geqq n_{0}(\varepsilon, M)$,

$$
\left\|\varphi_{t}^{n}\right\|_{-r_{0}}^{2} \leqq\left\|\varphi_{0}^{n}\right\|_{-r_{0}}^{2}+\left(C_{4}+1\right) t+\widetilde{Q}_{0, t}^{n}
$$

with $C_{4}$ independent of $M$ and $t<\tau_{M}^{n}$. Reasoning in the same way as in (6.38-40), we conclude from (7.17) and (7.21) that

$$
P^{n}\left\{\tau_{M}^{n} \leqq T\right\} \leqq 4 \varepsilon
$$

for $M$ large enough and $n \geqq n_{1}(\varepsilon, M)$. The modulus of continuity of $\varphi_{t}^{n}$ is shown to be uniform in probability in the same way as in (6.41-44). Thus, by (5.10-11), the sequence of processes $\xi_{t}^{n}$ is tight in $\Omega$. Of course, (6.46) also holds here. Thus, it only remains to identify the limit process of the critical fluctuations $\xi_{t}^{n}$.

Since $\psi_{t}^{n}$ converges to zero, we need only to investigate the limits of $\varphi_{t}^{n}$, which are clearly concentrated on the set of processes with values in $\mathbb{V}_{p_{0}}$, the linear space spanned by $\cos 2 \pi p_{0} \cdot x, \sin 2 \pi p_{0} \cdot x$. Let $f \in \mathscr{C}_{b}^{2}\left(\mathbb{R}^{2}\right), g_{j} \in \mathbb{V}_{p_{0}}$ for $j=1,2$, and set

$$
\tilde{f}(\mu)=f\left(\left\langle g_{1}, \mu\right\rangle,\left\langle g_{2}, \mu\right\rangle\right), \quad \mu \in \mathscr{C}^{\infty}(\mathbb{T})^{\prime} .
$$

Again, we may restrict ourselves to $t_{1}<t_{2}<\hat{\tau}$ with

and

$$
\hat{\tau}=\tau_{M}^{n} \wedge \inf \left\{t,\left\|\psi_{t}^{n}\right\|_{-r}^{2} \vee\left\|\left(\sigma_{t N^{1 / 2}}^{n}(\cdot)^{2}-\gamma^{\prime \prime}(0)\right) \lambda^{n}\right\|_{-r_{0}}^{2}>N^{1 / 4 \kappa} \alpha_{n}^{-1 / 2}\right\},
$$

$$
P^{n}\{\hat{\imath} \leqq T\} \leqq 6 \varepsilon \quad \text { for } \quad n \geqq n_{1}(\varepsilon, M) .
$$

Now, with $M_{t_{1}, t_{2}}^{f, n}$ from (6.51), we get

$$
\begin{aligned}
\tilde{f}\left(\varphi_{t_{2}}^{n}\right)= & \tilde{f}\left(\varphi_{t_{1}}^{n}\right)+\int_{t_{1}}^{t_{2}} \sum_{j} \widetilde{\partial_{j} f}\left(\varphi_{s}^{n}\right)\left[\gamma^{(4)}(0) / 3 ! \beta_{p_{0}}^{3}\left\langle g_{j},\left(\mathscr{J} * \varphi_{s}^{n}\right)^{3} \lambda^{n}\right\rangle+o_{M}(1)\right] d s \\
& +1 / 2 \int_{t_{1}}^{t_{2}} \sum_{i, j} \widetilde{\partial_{i j} f}\left(\varphi_{s}^{n}\right)[1+o(1)] 2 \gamma^{\prime \prime}(0)\left[\left\langle g_{i}, g_{j}\right\rangle+o_{M}(1)\right] d s+M_{t_{1}, t_{2}}^{f, n}
\end{aligned}
$$

Now

$$
\begin{aligned}
\left\langle g,\left(\mathscr{J} * \varphi^{n}\right)^{3} \lambda^{n}\right\rangle & =\sum_{q \in(\mathbb{Z} / n \mathbb{Z})^{d}} \widehat{g \hat{\lambda}^{n}}(q)\left(\widehat{\overline{\mathscr{J} * \varphi^{n}}}\right)^{3}(q) \\
& =\sum_{q_{1}, q_{2}, q_{3} \in\left\{ \pm p_{0}\right\}} \widehat{g \lambda^{n}}\left(q_{1}+q_{2}+q_{3}\right) \prod_{i=1}^{3} \overline{\hat{\mathscr{J}}}\left(q_{i}\right) \overline{\hat{\varphi}^{n}}\left(q_{i}\right)
\end{aligned}
$$




$$
\begin{aligned}
& =\hat{\mathscr{J}}^{3}\left(p_{0}\right)\left[3\left|\widehat{\varphi}^{n}\left(p_{0}\right)\right|^{2} 2 \operatorname{Re}\left(\widehat{g \lambda^{n}}\left(p_{0}\right) \widehat{\widehat{\varphi}^{n}}\left(p_{0}\right)\right)+2 \operatorname{Re}\left(\widehat{g \lambda^{n}}\left(3 p_{0}\right) \widehat{\varphi^{n} 3}\left(p_{0}\right)\right)\right] \\
& \left.=\hat{J}^{3}\left(p_{0}\right)\left[3\left|\varphi^{n}\left(p_{0}\right)\right|^{2}\left\langle g, \varphi^{n}\right\rangle+o_{M}(1)\right)\right] .
\end{aligned}
$$

Hence, by $(7.25,26)$, any limit process $\varphi_{t}$ of $\varphi_{t}^{n}$ satisfies the martingale problem

$$
\begin{aligned}
& \tilde{f}\left(\varphi_{t_{2}}\right)-\tilde{f}\left(\varphi_{t_{1}}\right)-\int_{t_{1}}^{t_{2}}\left[\sum_{k} \widetilde{\partial_{k} f}\left(\varphi_{s}\right) \gamma^{(4)}(0) /\left[2 \gamma^{\prime \prime}(0)^{3}\right]\left|\hat{\varphi}_{s}\left(p_{0}\right)\right|^{2}\left\langle g_{k}, \varphi_{s}\right\rangle\right. \\
& \left.\quad+1 / 2 \sum_{i, j} \widetilde{\partial_{i_{j}} f}\left(\varphi_{s}\right) 2 \gamma^{\prime \prime}(0)\left\langle g_{i}, g_{j}\right\rangle\right] d s,
\end{aligned}
$$

which is equivalent to (7.12). This completes the proof.

The unique invariant probability measure of the process $\hat{\varphi}_{t}\left(p_{0}\right) \in \mathbb{C}$ is

$$
v_{2}(d z)=\exp \left\{\gamma^{(4)}(0) /\left[16 \gamma^{\prime \prime}(0)^{4}\right]|z|^{4}\right\} d z / Z_{2}
$$

with normalization constant $Z_{2}$.

\section{Critical Fluctuations at a Triple Point}

Let us suppose that we are at a triple point where a ferromagnetic second-order phase transition and an antiferromagnetic one of frequency $p_{0}$ occur simultaneously. This means that

$$
\hat{\mathscr{J}}(0)=\hat{\mathscr{J}}\left(p_{0}\right)=\hat{\mathscr{J}}\left(-p_{0}\right)>0 \quad \text { and } \quad \hat{\mathscr{J}}(0)-\hat{\mathscr{J}}(q) \geqq \delta_{0}>0
$$

for all $q \in \mathbb{Z}^{d} \backslash\left\{0, \pm p_{0}\right\}$, and

$$
\beta_{0}=\left(\gamma^{\prime \prime}(0) \hat{\mathscr{J}}(0)\right)^{-1}=\left(\gamma^{\prime \prime}(0) \hat{\mathscr{J}}\left(p_{0}\right)\right)^{-1} .
$$

We continue to let assumptions (A1) and (A2) from Sect. 6 hold, with (7.3), i.e. $K_{0}=2$, like in the last section. The surviving component of the critical fluctuation process $\xi_{t}^{n}$ from (7.4) is now

$$
\begin{aligned}
& \mu_{t}^{n}(d x)=\hat{\xi}_{t}^{n}(0) \lambda^{n}(d x)+2\left[\operatorname{Re}\left(\hat{\xi}_{t}^{n}\left(p_{0}\right)\right) \cos \left(2 \pi p_{0} x\right)+\operatorname{Im}\left(\hat{\xi}_{t}^{n}\left(p_{0}\right)\right) \sin \left(2 \pi p_{0} x\right)\right] \lambda^{n}(d x), \\
& v_{t}^{n}(d x)=\xi_{t}^{n}(d x)-\mu_{t}^{n}(d x) .
\end{aligned}
$$

Theorem 7. Let (8.1-2), (A1) with (7.3), and (A2) from Sect. 6 hold. Assume

$$
\sigma_{0}^{n} \in \mathscr{M}_{b}^{n} \text {, and } \mu_{0}^{n} \text { converges in law to } \mu_{0} \text {; }
$$

(ii)

$$
\text { Equations (7.8) and (7.9) hold, together with }
$$

$$
E^{n}\left\|v_{t}^{n}\right\|_{-r_{0}}^{2 \kappa} \leqq C_{1} \alpha_{n}^{-\kappa}
$$

Then $\xi_{t}^{n}$ converges in law to the mixed-phase process

$$
\mu_{t}(d x)=\hat{\mu}_{t}(0) \lambda(d x)+2\left[\operatorname{Re}\left(\hat{\mu}_{t}\left(p_{0}\right)\right) \cos \left(2 \pi p_{0} x\right)+\operatorname{Im}\left(\hat{\mu}_{t}\left(p_{0}\right)\right) \sin \left(2 \pi p_{0} x\right)\right] \lambda(d x) .
$$

where $\left(\hat{\mu}_{t}(0), \hat{\mu}_{t}\left(p_{0}\right)\right)$ satisfies the coupled stochastic equation

$$
d \hat{\mu}_{t}(0)=\gamma^{(4)}(0) /\left[3 !\left(\gamma^{\prime \prime}(0)\right)^{3}\right]\left(\hat{\mu}_{t}(0)^{2}+6\left|\hat{\mu}_{t}\left(p_{0}\right)\right|^{2}\right) \hat{\mu}_{t}(0) d t+\left[2 \gamma^{\prime \prime}(0)\right]^{1 / 2} d w_{t},
$$




$$
d \hat{\mu}_{t}\left(p_{0}\right)=\gamma^{(4)}(0) /\left[2\left(\gamma^{\prime \prime}(0)\right)^{3}\right]\left(\hat{\mu}_{t}(0)^{2}+\left|\hat{\mu}_{t}\left(p_{0}\right)\right|^{2}\right) \hat{\mu}_{t}\left(p_{0}\right) d t+\left[2 \gamma^{\prime \prime}(0)\right]^{1 / 2} d w_{t}^{\complement}
$$

starting at $\left(\hat{\mu}_{0}(0), \hat{\mu}_{0}\left(p_{0}\right)\right)$, where $w_{t}$ and $w_{t}^{\mathbb{C}}$ are independent real, respectively complex-valued Brownian motions.

Proof. Again, we give only the main estimates and formulas, the arguments being the same as in the proof of Sect. 6 .

For $t_{1}<t_{2} \leqq \tau_{M}^{n}$, we have

with

$$
\begin{aligned}
\left\|v_{t_{2}}^{n}\right\|_{-r_{0}}^{2}= & \left\|v_{t_{1}}^{n}\right\|_{-r_{0}}^{2}+2 \int_{t_{1}}^{t_{2}}\left[N^{1 / 2}\left\langle v_{s}^{n}, \gamma^{\prime \prime}(0) \beta_{0} \mathscr{J} * v_{s}^{n} \lambda^{n}-v_{s}^{n}\right\rangle_{-r_{0}}+\mathcal{O}\left(\left\|v_{s}^{n}\right\|_{-r_{0}} M^{3}\right)\right. \\
& \left.+o(1)\left\|v_{s}\right\|_{-r_{0}}^{n} M\right] d s+\mathcal{O}(1)\left(t_{2}-t_{1}\right)+R_{t_{1}, t_{2}}^{n}
\end{aligned}
$$

$$
\begin{aligned}
R_{t_{1}, t_{2}}^{n}= & \int_{t_{1} N^{1 / 2}}^{t_{2} N^{1 / 2}} \int_{B \times T}\left[\| v_{s N_{-}^{-1 / 2}}^{n}+N^{-3 / 4}\left(m-\sigma_{s-}^{n}(x)\right)\left(\delta_{x}-\lambda^{n}-2 \cos \left(2 \pi p_{0}(x-\cdot)\right) \lambda^{n} \|_{-r_{0}}^{2}\right.\right. \\
& \left.-\left\|v_{s N_{-}^{-1 / 2}}^{n}\right\|_{-r_{0}}^{2}\right] \tilde{\Lambda}^{n}(d m, d x, d s) .
\end{aligned}
$$

This time, (7.15) holds for all $q \in(\mathbb{Z} / n \mathbb{Z})^{d} \backslash\left\{0, \pm p_{0}\right\}$; as above, we obtain

$$
\left\|v_{t_{2}}^{n}\right\|_{-r_{0}}^{2}=\left\|v_{t_{1}}^{n}\right\|_{-r_{0}}^{2}-\int_{t_{1}}^{t_{2}}\left(N^{1 / 2} \gamma^{\prime \prime}(0) \beta_{0} \delta_{0}\left\|v_{s}^{n}\right\|_{-r_{0}}+C_{3}(M)\right) d s+R_{t_{1}, t_{2}}^{n},
$$

with some martingale $R^{n}$; the proposition of the appendix yields

$$
P^{n}\left\{\sup _{t \leqq T \wedge \tau_{M}^{n}}\left\|v_{t}^{n}\right\|_{-r_{0}}^{2}>N^{1 / 4 \kappa} \alpha_{n}^{-1 / 2}\right\}<\varepsilon
$$

for all $n \geqq n_{0}(M, \varepsilon)$.

From the semi-martingale decomposition for $\left\|\mu_{t}^{n}\right\|_{-r_{0}}^{2}$, we derive estimates similar to $(7.18,19)$; combining the arguments of $(7.20)$ and of $(8.16)$ below, we prove $\gamma^{(4)}(0) / 3$ ! $\beta_{0}^{3}\left\langle\mu_{s}^{n},\left(\mathscr{I} * \mu_{s}^{n}\right)^{3} \lambda^{n}\right\rangle_{-r_{0}}<0$ : hence $\left\|\mu_{t}\right\|_{-r_{0}} \leqq\left\|\mu_{0}\right\|_{-r_{0}}+\left(C_{4}+1\right) t+$ $\widetilde{R}_{0, t}^{n}$, which implies, like in $(6.38-40), \quad P^{n}\left\{\tau_{M}^{n} \leqq T\right\} \leqq 4 \varepsilon$ for large $M$ and $n \geqq n_{1}(\varepsilon, M)$. $\mu_{t}^{n}$ is shown to have a modulus of continuity uniform in probability, such that by $(5.10-11) \xi_{t}^{n}$ is tight. The inequality (6.46) still holds. We only need to characterize the limits of $\xi_{t}^{n}$; they are continuous processes, with values in $\mathbb{V}_{3}$ the linear space spanned by $1, \cos 2 \pi p_{0} \cdot x$, and $\sin 2 \pi p_{0} \cdot x$. Let $f \in \mathscr{C}_{b}^{2}\left(\mathbb{R}^{3}\right), g_{k} \in \mathbb{V}_{3}$ for $j=1,2,3$, and define

$$
\tilde{f}(\mu)=f\left(\left\langle g_{1}, \mu\right\rangle,\left\langle g_{2}, \mu\right\rangle,\left\langle g_{3}, \mu\right\rangle\right), \quad \mu \in \mathscr{C}^{\infty}(\mathbb{T})^{\prime} .
$$

We define $\hat{\imath}$ as in (7.25) with $\psi_{t}^{n}$ replaced by $v_{t}^{n}$, such that (7.26) still holds, because of (8.13), and we get for $t_{1}<t_{2}<\hat{t}$,

$$
\begin{aligned}
\tilde{f}\left(\mu_{t_{2}}^{n}\right)= & \tilde{f}\left(\mu_{t_{1}}^{n}\right)+\int_{t_{1}}^{t_{2}} \sum_{j} \widetilde{\partial_{j} f}\left(\mu_{s}^{n}\right)\left[\left\langle g_{j}, \gamma^{(4)}(0) / 3 ! \beta_{0}^{3}\left(\mathscr{J} * \mu_{s}^{n}\right)^{3} \lambda^{n}\right\rangle+o_{M}(1)\right] d s \\
& +1 / 2 \int_{t_{1}}^{t_{2}} \sum_{i, j} \widetilde{\partial_{i j} f} f\left(\mu_{s}^{n}\right)(1+o(1)) 2 \gamma^{\prime \prime}(0)\left[\left\langle g_{i}, g_{j}\right\rangle+o_{M}(1)\right] d s+M_{t_{1}, t_{2}}^{f, n},
\end{aligned}
$$


with $M_{t_{1}, t_{2}}^{f, n}$ from (6.51) with $K_{0}=2$. We compute

$$
\begin{aligned}
\left\langle g,\left(\mathscr{J} * \mu_{s}^{n}\right)^{3} \lambda^{n}\right\rangle= & \sum_{q_{1}, q_{2}, q_{3} \in\left\{0, \pm p_{0}\right\}} \widehat{g \lambda^{n}}\left(q_{1}+q_{2}+q_{3}\right) \prod_{i=1}^{3} \overline{\hat{\mathscr{J}}}\left(q_{i}\right) \widehat{\hat{\mu}_{s}^{n}}\left(q_{i}\right) \\
= & g \lambda^{n}(0)\left[\hat{\mathscr{J}}(0) \widehat{\mu^{n 3}}(0)+6 \widehat{\mathscr{J}}(0) \hat{\mathscr{J}}^{2}\left(p_{0}\right) \mid \widehat{\mu^{n}}\left(p_{0}\right) \widehat{\mu^{n}}(0)\right] \\
& +6 \operatorname{Re}\left[\widehat{g \lambda^{n}}\left(p_{0}\right)\left(\hat{\mathscr{J}}^{2}(0) \hat{\mathscr{J}}\left(p_{0}\right) \widehat{\mu^{n 2}}(0) \widehat{\mu^{n}}\left(p_{0}\right)+\hat{\mathscr{J}}\left(p_{0}\right)\left|\widehat{\mu^{n}}\left(p_{0}\right)\right|^{2} \widehat{\mu^{n}}\left(p_{0}\right)\right)\right] \\
& +6 \operatorname{Re}\left[\widehat{g \lambda^{n}}\left(2 p_{0}\right) \mathscr{J}(0) \hat{\mathscr{J}}^{2}\left(p_{0}\right) \widehat{\mu^{n}}(0) \widehat{\mu^{n}}\left(p_{0}\right)\right] \\
& +2 \operatorname{Re}\left[\widehat{g \lambda^{n}}\left(3 p_{0}\right) \hat{\mathscr{J}}^{3}\left(p_{0}\right) \widehat{\mu^{n}}\left(p_{0}\right)\right] .
\end{aligned}
$$

Taking the limit $n \rightarrow \infty$, the last two terms vanish, and we get a limit process $\mu_{i}$ given by (8.7-9), since this is the only solution to the martingale problem

$$
\begin{aligned}
\tilde{f}\left(\mu_{t_{2}}\right)-\tilde{f}\left(\mu_{t_{1}}\right)= & \int_{t_{1}}^{t_{2}}\left[\sum_{j} \widetilde{\partial_{j} f}\left(\mu_{s}\right) \gamma^{(4)}(0) /\left[3 !\left(\gamma^{\prime \prime}(0)\right)^{3}\right]\left\{\left(\hat{\mu}_{s}^{2}(0)+6\left|\hat{\mu}_{s}\left(p_{0}\right)\right|^{2}\right) \hat{g}_{j}(0) \hat{\mu}_{s}(0)\right.\right. \\
& \left.+3\left(\hat{\mu}_{s}(0)^{2}+\left|\hat{\mu}_{s}\left(p_{0}\right)\right|^{2}\right)\left\langle g_{j}-\hat{g}_{j}(0), \mu_{s}\right\rangle\right\}+1 / 2 \sum_{i, j} \widetilde{\partial_{i j} f}\left(\mu_{s}\right) 2 \gamma^{\prime \prime}(0) \\
& \left.\cdot\left\{\hat{g}_{i}(0) \hat{g}_{j}(0)+2 \operatorname{Re}\left(\hat{g}_{i}\left(p_{0}\right) \overline{\hat{g}_{k}}\left(p_{0}\right)\right)\right\}\right] d s
\end{aligned}
$$

The proof of Theorem 7 is complete.

\section{Appendix. A Proposition on Collapsing Processes}

\section{Proposition}

(i) Let $X_{t}^{m} \geqq 0$ be a sequence of positive semimartingales with

$$
d X_{t}^{m}=S_{t}^{m} d t+\int f_{t-}^{m}(y)\left[\Lambda^{m}(d t, d y)-g_{t}^{m}(d y) d t\right] .
$$

Here $S_{t}^{m}$ and $f_{t}^{m}$ are adapted processes, $\Lambda^{m}$ is a point process on some measurable space $Y$ with compensator $g_{t}^{m}(d y) d t$. Let $\kappa>1$ and let $\alpha_{m}$ be an increasing sequence with

$$
\begin{gathered}
m^{1 / \kappa} \alpha_{m}^{-1} \rightarrow 0, \quad \alpha_{m} \cdot m^{-1} \rightarrow 0, \\
E^{m}\left(X_{0}^{m}\right)^{\kappa} \leqq C_{1} \alpha_{m}^{-\kappa} \quad \text { for all } m .
\end{gathered}
$$

Furthermore, $\tau^{m}$ are stopping times such that for $t \in\left[0, \tau^{m}\right], m \geqq 1$,

$$
\begin{gathered}
S_{t}^{m} \leqq-m \delta X_{t}^{m}+C_{2}, \quad \delta>0 \\
\sup _{\omega \in \Omega, y \in Y, t \leqq r^{n}}\left|f_{t}^{m}\right| \leqq C_{4} \alpha_{m}^{-1}, \\
\int_{Y}\left(f_{t}^{m}(y)\right)^{2} g_{t}(d y) \leqq C_{5} .
\end{gathered}
$$

(Here, and in the sequel, $C_{i}$ are constants independent of $m$ and $X_{t}^{m}$ ). Then for any $\varepsilon>0$, there exists $C_{6}>0$ and $m_{0}$ such that

$$
\sup _{m \geqq m_{0}} P^{m}\left\{\sup _{0 \leqq t \leqq T \wedge \tau^{m}} X_{t}^{m}>C_{6}\left(m^{1 / \kappa} \alpha_{m}^{-1} \vee \alpha_{m} m^{-1}\right)\right\} \leqq \varepsilon .
$$


(ii) If instead of (A.6), we have even

$$
\int_{Y}\left(f_{t}^{m}(y)\right)^{2} g_{t}(d y) \leqq C_{5}\left(X_{t}^{m}+m^{-1}\right)
$$

then we get instead of $(A .7)$,

$$
\sup _{m \geqq m_{0}} P^{m}\left\{\sup _{0 \leqq t \leqq T \wedge \tau^{m}} X_{t}^{m}>C_{6} m^{1 / \kappa} \alpha_{m}^{-1}\right\} \leqq \varepsilon .
$$

Proof. (We drop the superscript $m$ everywhere). Let $h$ be a smooth, positive, increasing, convex function on $\mathbb{R}$ with

and

$$
\left(y^{+}\right)^{\kappa} \leqq h(y) \leqq a+\left(y^{+}\right)^{\kappa},
$$

$$
\sup _{y_{1} \in \mathbb{B}\left|y_{2}\right| \leqq C_{4}} h^{\prime \prime}\left(y_{1}+y_{2}\right) / h^{\prime}\left(y_{1}\right)=C_{7}<\infty .
$$

Equation (A.11) implies $h\left(y_{1}+y_{2}\right)-h\left(y_{1}\right)-h^{\prime}\left(y_{1}\right) y_{2} \leqq 1 / 2 h^{\prime}\left(y_{1}\right) C_{7} y_{2}^{2}$ for all

$$
\left|y_{2}\right| \leqq C_{4} \text { and all } y_{1} \in \mathbb{R} \text {. }
$$

Now for $l=1, \ldots,[T m]+1$ and $t \leqq l / m \wedge T \wedge \tau$, let

$$
\begin{aligned}
Z_{i}^{l}= & \tilde{h}\left(X_{t}\right):=h\left(\alpha_{m} e^{\delta(m t-l)}\left(X_{t}-C_{2} / \delta m\right)-C_{7}\right. \\
& \left.\cdot \int_{0}^{t} \alpha_{m}^{2} e^{2 \delta(m s-l)} \int_{Y}\left|f_{s}(y)\right|^{2} g_{s}(d y) d s\right)
\end{aligned}
$$

Ito's formula gives

$$
\begin{aligned}
d Z_{t}^{l}= & \tilde{h}^{\prime}\left(X_{t}\right) \alpha_{m} e^{\delta(m t-l)}\left(m \delta X_{t}-C_{2}+S_{t}\right) \\
& +\int_{Y}\left[\tilde{h}\left(X_{t}+f_{t}\right)-\tilde{h}\left(X_{t}\right)-\tilde{h}^{\prime}\left(X_{t}\right)\left(\alpha_{m} e^{\delta(m t-l)} f_{t}+C_{7} \alpha_{m}^{2} e^{2 \delta(m s-l)}\left(f_{t}(y)\right)^{2}\right)\right] g_{t}(d y) \\
& +\int_{Y}\left[\tilde{h}\left(X_{t}+f_{t}\right)-\tilde{h}\left(X_{t}\right)\right]\left(\Lambda(d y, d t)-g_{t}(d y) d t\right) .
\end{aligned}
$$

Using (A.5), $m t-l \leqq 0$ and (A.12), the first two terms in (A.14) are non-positive, such that $Z_{t}^{l}$ are positive supermartingales on $t \leqq l / m \wedge T \wedge \tau$. Doob's inequality and (A.3) yield

$$
P\left(\bigcup_{l=1}^{[m T]+1}\left\{\sup _{t \leqq l / m \wedge T \wedge \tau^{m}} Z_{t}^{l}>m \eta^{-1}\right\}\right) \leqq m^{-1} \eta \sum_{l=1}^{[T m]+1} E\left(Z_{0}\right) \leqq \eta(T+1)\left(a+C_{1}\right) \leqq \varepsilon
$$

for $\eta$ sufficiently small. But $\sup Z_{t}^{l} \leqq m \eta^{-1}$ is equivalent to

$$
\alpha_{m} e^{\delta(m t-l)}\left(X_{t}-C_{2} / \delta m\right) \leqq h^{-1}\left(m \eta^{-1}\right)+C_{7} \int_{0}^{t} \alpha_{m}^{2} e^{2 \delta(m s-l)} \int_{Y}\left|f_{s}(y)\right|^{2} g_{s}(d y) d s
$$

for all $\iota \leqq l / m \wedge T \wedge \tau$. If we restrict $t$ to the interval $[l-1 / m, l / m \wedge T \wedge \tau]$, we see that by (A.10) and (A.6), respectively (A.8), (A.16) implies 


$$
\begin{aligned}
X_{t} \leqq & C_{2} \delta^{-1} m^{-1}+e^{\delta} h^{-1}\left(m \eta^{-1}\right) \alpha_{m}^{-1} \\
& +e^{\delta} C_{7} \alpha_{m} \sup _{5 \leqq t} \int_{Y}\left|f_{s}(y)\right|^{2} g_{s}(d y) e^{2 \delta(m t-l)}-e^{-2 \delta l} / 4 \delta m \\
\leqq & C_{2} \delta^{-1} m^{-1}+C_{8} m^{1 / k} \alpha_{m}^{-1}+C_{9} \alpha_{m} m^{-1}\left(\sup _{s \leqq t}^{1} X_{s}+m^{-1}\right),
\end{aligned}
$$

where the first component in the last bracket refers to the condition (A.6) and the second to (A.8). Thus by (A.2),

$$
\begin{aligned}
& \bigcap_{l=1}^{[m T]+1}\left\{\sup _{t \leqq l / m \wedge T \wedge \tau} Z_{t}^{l} \leqq m \eta^{-1}\right\} \subseteq\left\{\sup _{s \leqq t} X_{s} \leqq C_{2} \delta^{-1} m^{-1}+C_{8} m^{1 / K} \alpha_{m}^{-1}\right. \\
& \left.+C_{9} \alpha_{m} m^{-1}\left(\sup _{s \leqq t}^{1} X_{s}+m^{-1}\right)\right\} \\
& \subseteq\left\{\sup _{t \leqq T \wedge \tau} X_{s} \leqq C_{10}\left(\begin{array}{ll}
m^{1 / \mathscr{H}} & \alpha_{m}^{-1} \vee \alpha_{m} m^{-1} \\
m^{1 / \mathscr{K}} & \alpha_{m}^{-1}
\end{array}\right)\right\}
\end{aligned}
$$

for $m$ sufficiently large. (A.15) and (A.18) prove (A.7), respectively (A.9).

Acknowledgements. The first author would like to express his hearty thanks to R. Azencott for valuable discussions, and to M. Metivier for pertinent remarks.

Part of this work was done, while the second author was visiting the Courant Institute at New York University. He is grateful for its kind hospitality and the many useful discussions he had with its members, in particular with A. Sokal and S. R. S. Varadhan.

\section{References}

1. Comets, F.: Nucleation for a long range magnetic model. Ann. Inst. H. Poincaré 23, 2, 135-178 (1987)

2. Comets, F., Eisele, Th., Schatzman, M.: On secondary bifurcations for some nonlinear convolution equations. Trans. Am. Math. Soc. 296, 2, 661-702 (1986)

3. Dawson, D. A.: Critical dynamics and fluctuations for a meanfield model of cooperative behavior. $\mathbf{J}$. Stat. Phys. 31, 29-85 (1983)

4. Dawson, D. A., Gārtner, J.: Long-time fluctuations of weakly interacting diffusions, preprint

5. Dawson, D. A., Gārtner, J.: Large deviations from the McKean-Vlasov limit for weakly interacting diffusions, preprint 1986

6. Eisele, Th.: Equilibrium and nonequilibrium theory of a geometric long-range spin glass, Ecole d'été de Physique théorique. Les Houches 1984. Osterwaldet, K., Stora, R.: (eds). Amsterdam Oxford, New York: Elsevier Science 1986

7. Eisele, Th., Ellis, R. S.: Symmetry breaking and random waves for magnetic systems on a circle $Z$. Wahrsch. V. G. 63, 297-348 (1983)

8. Ellis, R. S., Monroe, J. L., Newman, C. M.: The GHS and other correlation inequalities for a classe of even ferromagnets. Commun. Math. Phys. 46, 167-182 (1976)

9. Ellis, R. S., Newman, C. M., Rosen, J. S.: Limit theorems for sums of dependent random variables occurring in statistical mechanics. Z. Wahrsch. Verw. Geb. 51, 153-169 (1980)

10. Fritz, J.: The Euler equation for the stochastic dynamics of a one-dimensional continuous spin system. Preprint 1986

11. Glauber, R. J.: Time-dependent statistics of the Ising model. J. Math. Phys. 4, 294 (1963)

12. Hida, T.: Brownian Motion. Berlin, Heidelberg, New York, 1980

13. Holley, R. A., Stroock, D. W.: Generalized Ornstein-Uhlenbeck processes and infinite particle branching Brownian motions. Res. Inst. Math. Sci. Kyoto 14, 741-788 (1978) 
14. Ikeda, N., Watanabe, S.: Stochastic differential equations and diffusion processes. Amsterdam: North-Holland 1981

15. Ito, K.: Foundations of stochastic differential equations in infinite dimensional spaces. Philadelphia: Soc. Ind. Appl. Math. 1984.

16. Jacod, J.: Théorèmes limite pour les processus, Ecole d'été de probabilités de St Flour XIII-1983. Lecture Notes in Mathematics, Vol. 1117, Berlin, Heidelberg, New York: Springer 1985

17. Kipnis, C.: Processus de champ moyen. Stochastics 5, 93-106 (1981)

18. McKean, H. P.: Propagation of chaos for a class of nonlinear parabolic equations, Lect. Ser. Diff. Eq. 2, 41-57 New York: Van Nostrand Reinhold 1969

19. McKean, H. P.: Fluctuations in the kinetic theory of gases. Commun. Pure Appl. Math. 28, 435-455 (1975)

20. Mitoma, I.: Tightness of probabilities on $\mathscr{C}\left([0,1], \mathscr{S}^{\prime}\right)$ and $\mathscr{D}\left([0,1], \mathscr{S}^{\prime}\right)$. Ann. Prob. 11, 989-999 (1983)

21. Nagasawa, M., Tanaka, H.: Propagation of chaos for diffusing Particles of two types with singular mean field interaction. Prob. Th. Rel. F. 71, 69-83 (1986)

22. Oelschlāger, K.: A martingale approach to the law of large numbers for weakly interacting stochastic processes. Ann. Prob. 12, 458-479 (1984)

23. Shiga, T., Tanaka, H.: Central limit theorems for a system of Markovian particles with mean field interactions. Z. Wahrsch. Verw. Geb. 69, 439-459 (1985)

24. Strichartz, R. S.: Multipliers on fractional Sobolev Spaces. J. Math. Mech. 16, 1031-1060 (1967)

25. Sznitman, A. S.: Nonlinear reflecting dffusion process, and the propagation of chaos and fluctuations associated. J. Funct. Anal. 56, 311-336 (1984)

26. Tanaka, H. Histsuda, M.: Central limit theorem for a simple diffusion model of interacting particles. Hiroshima Math. J. 11, 415-423 (1981)

27. Spitzer, F.: In: St Flour 73, Lecture Notes in Mathematics, Vol. 390. Berlin, Heidelberg, New York: Springer 1974

28. Meyer, Y.: In Séminarie Bourbaki 1979-1980, $n^{\circ} 560$ (1980)

29. Bers, L., John, F., Schechter, M.: Partial differential equations. New York: Intersciences 1964

Communicated by J. L. Lebowitz

Received January 30, 1987; in revised form March 29, 1988 
